\title{
NEARLY KÄHLER GEOMETRY AND RIEMANNIAN FOLIATIONS *
}

\author{
PAUL-ANDI NAGY ${ }^{\dagger}$
}

\begin{abstract}
We consider strict and complete nearly Kähler manifolds with the canonical Hermitian connection. The holonomy representation of the canonical Hermitian connection is studied. We show that a strict and complete nearly Kähler is locally a Riemannian product of homogenous nearly Kähler spaces, twistor spaces over quaternionic Kähler manifolds and 6-dimensional nearly Kähler manifolds. As an application we obtain structure results for totally geodesic Riemannian foliations admitting a compatible Kähler structure. Finally, we obtain a classification result for the homogenous case, reducing a conjecture of Wolf and Gray to its 6-dimensional form.
\end{abstract}

1. Introduction. Given an oriented Riemannian manifold $\left(M^{n}, g\right)$ a fundamental piece of data is encoded in its holonomy group, to be denoted by $\operatorname{Hol}(M, g)$. Indeed, the condition that $\operatorname{Hol}(M, g)$ be different from $S O(n)$ has strong geometric implications at the level of the geometric structure supported by $M$ (see [31] for an account). The search for new geometric structures supporting (for example) Einstein metrics or satisfying usefull curvature identities motivated the introduction by A. Gray of the concept of weak holonomy (see [12] and [32]). However, in the case when the weak holonomy group of a manifold acts transitvely on its unit bundle it was shown [12] that only three groups, namely

$$
U(n), G_{2} \text { and } \operatorname{Spin}(9)
$$

can potentially produce new geometric structures (other than those coming from Riemannian holonomy). This is certainly the case for weak holonomy $G_{2}$ (see [8]), where many homogenous examples were constructed. Also, it is now known that every 7 dimensional 3-sasakian manifold carry a metric with weak holonomy $G_{2}$ and, furthermore, there is no scarcity of such manifolds (see [3]). Very recentely, the case of $\operatorname{Spin}(9)$ was undertaken by Friedrich [9].

In this paper we will be concerned with the study of manifolds with weak holonomy $U(n)$. These manifolds are called nearly Kähler and also appear as one of the sixteen classes of almost Hermitian manifolds [15]. Many properties of this class of manifolds are now known (see $[11,13,14]$ ). Note the 6-dimensional case stands out because of the existence of Killing spinors (fact that is in this dimension equivalent to being nearly Kähler [16]) and because of carrying Einstein metrics.

Nearly Kähler manifolds can also be caractherized as almost Hermitian manifolds admitting a metric Hermitian connection whose torsion is parallel and totally skew. From this point of view they are interesting in theoretical physics [10]. Manifolds carrying a connection with totally skew-symmetric, parallel, torsion were used in [5], as a point of departure to propose a framework somewhat parallel to weak holonomy, formulated in terms of $G$-structures (see [35] for a brief account). The main result of our paper is a classification result of nearly Kähler manifolds, given below.

THEOREM 1.1. Let $\left(M^{2 n}, g, J\right)$ be a strict and complete, simply connected, nearly Kähler manifold. Then $M$ is a Riemannian product whose factors belong to one of the following three classes:

- 6 dimensional nearly Kähler manifolds;

\footnotetext{
*Received May 24, 2002; accepted for publication July 12, 2002.

†Institut de Mathématiques, rue E. Argand 11, 2007 Neuchâtel, Switzerland (Paul.Nagy@unine.ch).
} 
- homogenous nearly Kähler spaces of type I,II,III or IV;

- twistor spaces over quaternionic Kähler manifolds with positive scalar curvature, endowed with the canonical nearly Kähler metric

Of course, the previous decomposition coincides with the deRham decomposition of the manifold $(M, g)$. The homogenous spaces of type I and II and described in sections 2 and 3 and those of types III and IV in sections 5 and 6 . The latter are generalized "twistor spaces", that is Riemannian submersions whose (totally geodesic) fibers are compact and simply connected Hermitian symmetric spaces and whose base spaces are compact and simply connected symmetric spaces.

Hence, weak holonomy $U(n)$ does not produce any new geometric structure, except possibly in real dimension 6 . But even in this case, the only known examples are homogenous.

The proof of our theorem goes as follows. In view of the results of [5] we are mainly concerned with the case when the holonomy of the canonical Hermitian connection is reducible. Then, our point of departure is to show that one can suppose that the holonomy representation is of special algebraic type, that is it has strong algebraic properties related to the torsion tensor of the Hermitian connection. This will be done in section 3 . In the case of special algebraic torsion, we show in section 4 that the manifold $M$ is the total space of a fibration whose (totally geodesic ) fibers are compact and simply connected Hermitian symmetric spaces. It turns out that for such fibrations we can prove an analogue of the DeRham decomposition theorem, in the sense the one can always suppose that the fiber and the base space are irreducible, in the usual Riemannian sense. After develloping some facts related to the Ricci tensor in section 5 we finally prove the classification result in section 6 . It reposes on the observation that the torsion tensor points out in a nice way to the Riemannian holonomy group of the base manifold, hence allowing the use of the Berger classification theorem.

As a corollary of theorem 1.1 we show how one can use the classification of nearly Kähler manifolds in order to study geometric structures on Kähler manifolds. To cite some related results, recall that it was known for a long time that that a Kähler submersion has integrable and totally geodesic horizontal distribution, hence zero curvature [37, 18]. The same conclusion holds for nearly Kähler and almost Kähler submersions [7]. In twistor theory, one searches for a non-holomorphic fibration whose total space and fibers are complex manifolds. A reasonable way to see these objets arise is from Riemannian foliations. Unfortunately, the following result shows that it cannot happen in the presence of a Kähler metric, except for a few special cases.

THEOREM 1.2. Let $(M, g, J)$ be a simply connected Kähler manifold supporting a foliation $\mathcal{F}$ such that $(M, g, \mathcal{F})$ becomes a totally geodesic Riemannian foliation. If the leaves of $\mathcal{F}$ are complex manifolds then $(M, g)$ is a Riemannian product whose factors belong to one the following three classes:

- twistor spaces over quaternionic Kähler manifolds of positive scalar curvature ;

- compact homogenous Kähler manifolds corresponding to nearly Kähler manifolds of types III and IV;

- Kähler manifolds.

Furthermore, the foliation $\mathcal{F}$ is obtained in a canonical way from the above decomposition.

This is not a very surprising result, as geometric structures on Kähler manifolds are rather rare. As an example which parallels theorem 1.2 we cite the result that a complex contact manifold with a Kähler-Einstein metric is the twistor space of a 
quaternionic Kähler manifold of positive sectional curvature [23]. The main ingredient of the proof of the theorem 1.2, given in section 7 , is the observation that Kähler manifolds admitting a Riemannian foliation as above have a canonical nearly Kähler metric. Next we use the results leading to the proof of theorem 1.1.

Finally, in section 8 we study homogenenous nearly Kähler manifolds using theorem 1.1. A classification result involving the previously defined spaces of type $I, I I, I I I$ and $I V$ is obtained. As a consequence, we recover by geometric means the classification of naturally reductive Riemannian 3-symmetric spaces, which was obtained in his general form in [13] using Lie theory. We are also able to reduce a conjecture of Wolf and Gray relating to nearly Kähler homogenenous spaces to its 6-dimensional version.

Acknowledgments. The author wishes to thank Andrew Swann for usefull discussions during the preparation of this work and also Alain Valette for explanations on representation theory.

2. Preliminaries. A nearly Kähler manifold is an almost Hermitian manifold $\left(M^{2 n}, g, J\right)$ such that

$$
\left(\nabla_{X} J\right) X=0
$$

for every vector field $X$ on $M$ (here $\nabla$ denotes the Levi-Civita connection associated to metric $g$ ). It is called strict if $\nabla_{X} J \neq 0$ whenever $X \in T M, X \neq 0$.

Recall that the tensor $\nabla J$ has a number of important algebraic properties that can be summarized as follows : the tensors $A$ and $B$ defined for $X, Y, Z$ in $T M$ by $A(X, Y, Z)=<\left(\nabla_{X} J\right) Y, Z>$ and $B(X, Y, Z)=<\left(\nabla_{X} J\right) Y, J Z>$ are skew-symmetric and have type $(0,3)+(3,0)$ as real 3 -forms. Another object of particular importance is the canonical Hermitian connection defined by

$$
\bar{\nabla}_{X} Y=\nabla_{X} Y+\frac{1}{2}\left(\nabla_{X} J\right) J Y
$$

It is easy to see that $\bar{\nabla}$ is the unique Hermitian connection on $M$ with totally skew-symmetric torsion (see for example [10]). Note that the torsion of $\bar{\nabla}$ given by $T(X, Y)=\left(\nabla_{X} J\right) J Y$ vanishes iff $(M, g, J)$ is a Kähler manifold.

Let us define $\Phi \in \Lambda^{3}(M)$ by $\Phi(X, Y, Z)=<\left(\nabla_{X} J\right) Y, Z>$ whenever $X, Y, Z$ are in $T M$. A fact of crucial importance for us will be that the form $\Phi$ is parallel with respect to the canonical Hermitian connection, that is :

$$
\bar{\nabla} \Phi=0 .
$$

This can be deduced from the $\bar{\nabla}$ parallelism of the tensor $\nabla J$ which was proven in [1]. This particularly implies that the holonomy group of $\bar{\nabla}$ is contained at each point in the $U(n)$-stabiliser of the three form $\Phi$. The following decomposition result shows that one can restrict attention to strict nearly Kähler manifolds.

Proposition 2.1. [19, 24] Let $(M, g, J)$ be a complete and simply connected nearly Kähler manifold. Then $M$ is a Riemannian product $M_{1} \times M_{2}$ where $M_{1}$ and $M_{2}$ are Kähler respectively strict nearly Kähler manifolds.

We discuss now briefly some properties of the Ricci tensor of nearly Kähler manifolds. Recall that it was shown in [24] that if $(M, g, J)$ is strict then it has positive 
Ricci curvature, hence $(M, g, J)$ is compact as soon as it is complete. Moreover, under these conditions the fundamental group is finite hence the requirement of simple connectivity (to be done in most cases in this work) is not too restrictive. Now, the Ricci- $\star$ curvature is defined by :

$$
\operatorname{Ric}^{\star}(X, Y)=\frac{1}{2} \sum_{i=1}^{2 n} R\left(X, J Y, e_{i}, J e_{i}\right)
$$

where $R$ is the curvature tensor of $(M, g)$ and $\left\{e_{1}, \ldots, e_{2 n}\right\}$ a local frame field. The difference of the Ricci and Ricci- $\star$ curvature tensors, to be denoted by $r$, is given by the formula (see [14]) :

$$
<r X, Y>=\sum_{i=1}^{2 n}<\left(\nabla_{e_{i}} J\right) X,\left(\nabla_{e_{i}} J\right) Y>
$$

Then $r$ is symmetric, positive and commutes with $J$; furthermore we have $\bar{\nabla} r=0$ (see [24]). It is well known [14] that if the tensor $r$ has a single eigenvalue then $(M, g)$ is an Einstein manifold and furthermore the first Chern class of $(M, J)$ vanishes.

From now on we will work with a strict and complete nearly Kähler manifold $\left(M^{2 n}, g, J\right)$. The torsion tensor of the canonical Hermitian connection can be used to define a vector product on $T M$ by setting

$$
X \bullet Y=T(X, Y)
$$

whenever $X, Y$ belong to $T M$. The algebraic properties of the torsion tensor $T$ translate into analogous ones for the previous defined vector product. If $V, W$ are distributions of $T M$ we will usually denote by $V \bullet W$ the linear span of $\{x \bullet y: x \in V, y \in W\}$.

Let us recall that the first Chern class of $(M, J)$ is represented by the closed 2form $\gamma_{1}$ defined by $8 \pi \gamma_{1}(X, Y)=\langle C X, J Y>$ for all $X, Y$ in $T M$. Here $C$ denotes the symmetric endomorphism Ric-5Ric . It has been proven in [24] that the tensor $C$ is $\bar{\nabla}$-parallel. As $\gamma_{1}$ is a closed form we obtain easily

$$
-C(X \bullet Y)=X \bullet C Y+C X \bullet Y
$$

for all $X$ and $Y$ in $T M$.

REMARK 2.1. A basic fact about $C$ is that if $E_{i}$ and $E_{j}$ are eigenspaces of $C$ with corresponding eigenvalues $\lambda_{i}$ and $\lambda_{j}$ then $-\left(\lambda_{i}+\lambda_{j}\right)$ is an eigenvalue of $C$ if $E_{i} \bullet E_{j} \neq 0$.

Proposition 2.2. Suppose that $M$ is simply connected. If $C$ has a single eigenvalue then it splits as a Riemannian product whose factors are strict nearly Kähler manifolds such that their corresponding tensors $C$ and $r$ have exactly one eigenvalue.

Proof. For the inverse implication see [14]. Let us suppose now that $C$ has a single eigenvalue. Then $C=0$ by $(2.2)$ and more, Ric $=\frac{5}{4} r$. We recall now the expression of the Ricci curvature of $(M, g)$ we obtained in [24]. If $V_{i}, 1 \leq i \leq p$ are the eigenspaces of $r$ corresponding to the eigenvalue $\lambda_{i}$ then

$$
\operatorname{Ric}(X, Y)=\frac{\lambda_{i}}{4}\langle X, Y\rangle+\frac{1}{\lambda_{i}} \sum_{s=1}^{p} \lambda_{s}\left\langle r^{s}(X), Y\right\rangle
$$


for all $X$ and $Y$ in $V_{i}$, where the tensors $r^{s}, 1 \leq s \leq p$ are defined by $\left\langle r^{s}(X), Y>=\right.$ $-\operatorname{Tr}_{V_{s}}\left(\nabla_{X} J\right)\left(\nabla_{Y} J\right)$. Let us now suppose that the eigenvalues of $r$ are ordered as follows : $0<\lambda_{1}<\ldots<\lambda_{p}$. Then, on $V_{p}$ we must have $\lambda_{p}=\frac{1}{\lambda_{p}} \sum_{s=1}^{p} \lambda_{s} r^{s}$. As $r=\sum_{s=1}^{p} r^{s}$ this implies easily that $r^{s}=0,1 \leq s \leq p-1$ on $V_{p}$. In other words, $V_{p} \bullet H=0$ where $H=\bigoplus_{s=1}^{p-1} V_{s}$ and it follows that $V_{p} \bullet V_{p} \subseteq V_{p}$ and $H \bullet H \subseteq H$. Hence, the distributions $\stackrel{s=1}{V_{p}}$ and $H$ are $\nabla$-parallel, thus -by DeRham's decomposition theorem- $M$ is a Riemannian product $M_{1} \times M_{2}$. It easy to show that $M_{1}, M_{2}$ are strict nearly Kähler manifolds and $M_{1}$ has the property that the corresponding tensors $r$ and $C$ have exactly one eigenvalue. Now we apply the same procedure to the manifold $M_{2}$ and we conclude by induction.

REMARK 2.2. (i) Note that a result analogous to proposition 2.1 was already proven by different means in [36] under the assumptions of Riemannian irreducibility and constant scalar curvature. Note that the latter is now known to hold for all strict nearly Kähler manifolds. By the work in [33], proposition 2.1 remains true if we replace the vanishing of the Chern form with the vanishing of the Chern class.

(ii) Manifolds with $C=0$ and $r$ with a single eigenvalue are automatically Einstein.

In the rest of this section we will set up some facts concerning the holonomy representation of the canonical Hermitian connection. We begin with the following elementary result in representation theory. We also include its proof as we were unable to find it in the litterature.

LEMMA 2.1. Let $\left(V^{2 n}, g, J\right)$ a real vector space equipped with a scalar product $g$ and a compatible complex structure $J$. Let $V^{\mathbb{C}}$ the complex vector space obtained from $V$ by defining the complex multiplication to be $i . v=J v$. Let $\pi$ be a real representation of a group $G$ on $V$ respecting the inner product and the complex structure. Let $\pi^{\mathbb{C}}$ be the complex representation of $G$ on $V^{\mathbb{C}}$ induced by $\pi$. If $\pi^{\mathbb{C}}$ is irreducible then the following possibilities may occur :

(i) $\pi$ is irreducible;

(ii) there exists an irreducible $G$-submodule of $V$, to be denoted by $\mathcal{V}$, such that $V=$ $\mathcal{V} \oplus J \mathcal{V}$, an orthogonal direct sum.

Proof. If $\pi$ is irreducible then we are in the case of (i). Suppose now that the representation of $G$ on $V$ is real reducible. Let $\mathcal{V}$ be a proper invariant subspace. Then $\mathcal{V} \cap J \mathcal{V}$ is preserved by $J$ and $G$-invariant so it follows that $\mathcal{V} \cap J \mathcal{V}=0$. The same argument implies that we have the direct sum decomposition $V=\mathcal{V} \oplus J \mathcal{V}$. We will show now that the representation of $G$ on $\mathcal{V}$ is irreducible. Indeed, if $E \subseteq \mathcal{V}$ is non-zero $G$ module then as before $E \cap J E=0$ and $E \oplus J E=V$, hence $E$ and $\mathcal{V}$ have the same dimension and thus $E=\mathcal{V}$. It remains to show that the sum $\mathcal{V} \oplus J \mathcal{V}$ is orthogonal. Using Riesz's representation theorem we obtain the existence of a skew-symmetric endomorphism of $\mathcal{V}_{m}$, to be denoted by $F$, such that :

$$
<v, J w>=<F v, w>\text { for all } v, w \text { in } \mathcal{V} .
$$

Of course, $F$ must be $G$-invariant. We extend $F$ to a endomorphism $f$ of $V$, by setting $f(J v)=J(F v)$ for all $v$ in $\mathcal{V}$. Obviously this is complex linear and $G$-invariant, so using the irreducibility of $V^{\mathbb{C}}$ and Schur's lemma we obtain that $f=z 1_{V^{\mathbb{c}}}$ for some complex number $z$. The definition of $f$ and the skew-symmetry of $F$ imply now the vanishing of $F$. $\square$ 
Before applying this to our geometric context let us recall the following important result.

TheOREM 2.1. [5] Let $\left(M^{n}, g\right)$ be a simply connected Riemannian manifold equipped with a metric connexion $\nabla^{\prime}$ whose torsion tensor, denoted by $T$, is $\nabla^{\prime}$-parallel and totally skew. If the Lie algebra of the stabiliser of $T$ in $\mathfrak{s o}(n)$ acts irreducibly on some tangent space, then $M$ is a homogenous space with irreducible isotropy unless $S t a b_{\mathfrak{s o}(n)} T$ equals $G_{2}$ or $S U(3)$ acting as usually on $\mathbb{R}^{7}$ and $\mathbb{R}^{6}$ respectively.

REMARK 2.3. The two exceptions in theorem 2.1 are weak holonomy structures, precisely nearly parallel $G_{2}$ and six dimensional nearly Kähler manifolds. The basic ingredient for the proof of the previous result is a structure theorem for the space of algebraic curvature tensors of a Lie algebra preserving a three form. The homogenous structure on $M$ is obtained by proving that $\nabla^{\prime}$ is an Ambrose-Singer connexion, that is $\nabla^{\prime} R^{\prime}=0$. Also, it would be interesting to have a differential geometric proof of this result.

This remark motivates the setting, in the nearly Kähler case, of the following definition.

Definition 2.1. A strict nearly Kähler manifold $\left(M^{2 n}, g, J\right)$ with $n \geq 4$ is said homogenous of type $I$ if the holonomy representation of the canonical Hermitian connection is real irreducible.

By the previous discussion, in this case $\bar{\nabla}$ is a Ambrose-Singer connexion and moreover, $M$ is isotropy irreducible. We can now use the previous preliminaries in order to distinguish nearly Kähler manifolds by holonomic means, as follows.

Proposition 2.3. Let $\left(M^{2 n}, g, J\right)$ a strict, complete and simply connected nearly Kähler manifold. Then the following possibilities may occur:

(i) $M$ is homogenous of type $I$, unless $M$ is of dimension 6 ;

(ii) we have a $\bar{\nabla}$-parallel, orthogonal, decomposition $T M=\mathcal{V} \oplus H$ with $H=J \mathcal{V}$;

(iii) the holonomy representation of the canonical Hermitian connection is complex reducible.

Proof. We use that the torsion of the canonical Hermitian connection is parallel and totally skew. In particular, this implies that if the holonomy representation of $\bar{\nabla}$ is real irreducible then the same is true for representation of the $\mathfrak{s o}(2 n)$-stabilizer of the three form $\Phi$. We conclude by lemma 2.1 and theorem 2.1 .

Notice the difference with the classical Riemannian case, where there is no scarcity of holonomy irreducible structures. We mention apart the coresponding version of proposition 2.3 in six dimensions. We first set first the following

DEFINITION 2.2. Let $(M, g, J)$ be a 6-dimensional nearly Kähler manifold. It is called Hermitian irreducible if the holonomy representation of the canonical Hermitian connexion is irreducible in the real sense (and hence the Hermitian holonomy equals $S U(3))$.

Corollary 2.1. Let $(M, g, J)$ be a complete and simply connected nearly Kähler 6-fold. The following cases may occur :

(i) $(M, g, J)$ is Hermitian irreducible;

(ii) we have a $\bar{\nabla}$-parallel, orthogonal, decomposition $T M=\mathcal{V} \oplus H$ with $H=J \mathcal{V}$;

(iii) $(M, g, J)$ is one of the spaces $P \mathbb{C}^{3}, \mathbb{F}^{3}$ equipped with its canonical nearly Kähler metric.

Proof. Follows immediately from proposition 2.3 and [1] (see also [24]). 
Thus, in order to achieve the classification of strict nearly Kähler manifolds it remains to treat the cases when the holonomy representation of the canonical Hermitian connection is reducible, first in the real sense precised at the point (ii) of the proposition 2.3 and next in the general complex sense. We leave open the problem of classifying Hermitian irreducible nearly Kähler 6-folds.

3. Curvature properties and reducibility. In this section we will consider a strict nearly Kähler manifold $\left(M^{2 n}, g, J\right)$ which is reducible in the real Hermitian sense, that is the tangent bundle of $M$ admits a $\bar{\nabla}$-parallel, orthogonal, decomposition:

$$
T M=\mathcal{V} \oplus H
$$

In the first part of this section we will show that it is always possible to suppose that the previous decomposition is of special type, that is $\mathcal{V} \bullet \mathcal{V} \subseteq \mathcal{V}$. This will be done by a carefull examination of the curvature tensor of the canonical Hermitian connection, to be denoted by $\bar{R}$. Some preliminaries are required.

Lemma 3.1. Let $X$ be in $H, Y$ in $T M$ and $V, W$ in $\mathcal{V}$. We have :

$$
\bar{R}(X, Y, V, W)=<\left[\nabla_{V} J, \nabla_{W} J\right] X, Y>-<\left(\nabla_{X} J\right) Y,\left(\nabla_{V} J\right) W>.
$$

Proof. Let us recall the relation (see [14] page 237):

$$
\begin{aligned}
& \bar{R}(X, Y, Z, T)=R(X, Y, Z, T)-\frac{1}{2}<\left(\nabla_{X} J\right) Y,\left(\nabla_{Z} J\right) T>+ \\
& \left.\frac{1}{4}\left[<\left(\nabla_{X} J\right) Z,\left(\nabla_{Y} J\right) T>-<\left(\nabla_{X} J\right) T,\left(\nabla_{Y} J\right) Z\right\rangle\right]
\end{aligned}
$$

where $R$ denotes the curvature tensor of the Levi-Civita connection of $g$. It follows by the first Bianchi identity that

$$
R\left(X, Y, V_{1}, V_{2}\right)+R\left(Y, V_{1}, X, V_{2}\right)+R\left(V_{1}, X, Y, V_{2}\right)=0 .
$$

Or $\bar{R}\left(Y, V_{1}, X, V_{2}\right)=0$ so by (3.1) we get

$$
\begin{aligned}
& R\left(Y, V_{1}, X, V_{2}\right)=\frac{1}{2}<\left(\nabla_{Y} J\right) V_{1},\left(\nabla_{X} J\right) V_{2}> \\
& -\frac{1}{4}\left[<\left(\nabla_{Y} J\right) X,\left(\nabla_{V_{1}} J\right) V_{2}>-<\left(\nabla_{Y} J\right) V_{2},\left(\nabla_{V_{1}} J\right) X>\right] .
\end{aligned}
$$

In the same manner we obtain an analogous formula for $R\left(V_{1}, X, Y, V_{2}\right)$ and the result follows easily. $\square$

Corollary 3.1. Let $\left(M^{2 n}, g, J\right)$ be complete, strict and simply connected nearly Kähler manifold satisfying the conditions of (ii) in proposition 2.3. Then $(M, g)$ is a homogenous space.

Proof. Let $V_{i}, 1 \leq i \leq 4$ be in $\mathcal{V}$. Then using lemma 3.1 we get

$$
\bar{R}\left(V_{1}, V_{2}, J V_{3}, J V_{4}\right)=<\left[\nabla_{V_{1}} J, \nabla_{V_{2}} J\right] V_{3}, V_{4}>+<\left(\nabla_{V_{1}} J\right) V_{2},\left(\nabla_{V_{3}} J\right) V_{4}>.
$$

Using now that $\bar{R}\left(V_{1}, V_{2}, J V_{3}, J V_{4}\right)=\bar{R}\left(V_{1}, V_{2}, V_{3}, V_{4}\right)=\bar{R}\left(J V_{1}, J V_{2}, J V_{3}, J V_{4}\right)$ it follows that (3.2) completely determines the curvature $\bar{R}$ on $T M$. We recall now that $\bar{\nabla}(\nabla J)=0$, a fact proven in [1], which is equivalent to

$$
\bar{\nabla}_{U}\left(\left(\nabla_{X} J\right) Y\right)=\left(\nabla_{X} J\right) \bar{\nabla}_{U} X+\left(\nabla_{\bar{\nabla}_{U} X} J\right) Y
$$


whenever $U, X, Y$ are in $T M$. Thus, derivating formulas of type (3.2) we get easily that $\bar{\nabla} \bar{R}=0$, hence $\bar{\nabla}$ is an Ambrose-Singer connection. As $M$ is simply connected, we have that $(M, g, J)$ is a homogenous space (see [28]).

It is convenient to distinguish now a second class of homogenous nearly Kähler manifolds as follows.

DEFINITION 3.1. A simply connected, strict, nearly Kähler manifold is homogenous of type II iff the holonomy representation of $\bar{\nabla}$ on $T_{m}^{\mathbb{C}}(M)$ is complex irreducible and satisfies the conditions of (ii) in proposition 2.3.

REMARK 3.1. (i) In section 8 we will study in more detail the class of nearly Kähler manifolds having the property that $\bar{\nabla}$ is an Ambrose-Singer connection.

(ii) A typical example of nearly Kähler manifold of type II is provided by products $G \times G$ where $G$ is a compact Lie group without center (see [13]). However, it is not straightforward to see that these examples exhaust our class of spaces. This problem is studied in [25].

Hence, when the holonomy representation of the canonical Hermitian connexion is complex irreducible, $(M, g, J)$ is homogeneous of type I or II. Therefore, it remains to understand the complex reducible case. In this situation, using parallel transport we get a $\bar{\nabla}$-parallel decomposition $T M=\mathcal{V} \oplus H$ which is stable by $J$. For this reason, all the distributions we will consider from now on will be preserved by $J$.

Now, let us state and prove the following important consequence of lemma 3.1.

Corollary 3.2. Let $X, Y$ be in $H$ and $V, W$ be in $\mathcal{V}$ respectively. Then :

(i) $\left(\nabla_{X} J\right)\left(\nabla_{V} J\right) W=0$;

(ii) $\left(\nabla_{X} J\right)\left(\nabla_{Y} J\right) Z$ belongs to $H$ whenever $Z$ belongs to $H$;

(iii) $\left(\nabla_{V} J\right)\left(\nabla_{W} J\right) X$ belongs to $H$;

(iv) $\left(\nabla_{X} J\right)\left(\nabla_{Y} J\right) V$ belongs to $\mathcal{V}$.

Proof. (i) We use that $\bar{R}(J X, J Z, V, W)=\bar{R}(X, Z, V, W)$ for all $Z$ in $T M$, lemma 3.1 and the algebraic properties of the tensor $\nabla J$ (see Section 2).

(ii) Reversing the roles of $\mathcal{V}$ and $H$ we obtain that $\left(\nabla_{V} J\right)\left(\nabla_{X} J\right) Y=0$ for all $X, Y$ in $H$ and $V$ in $\mathcal{V}$. Taking the scalar product with $Z$ in $H$ gives now the result.

(iii) We choose $Y=V_{1}$ in lemma 3.1, where $V_{1}$ belongs to $\mathcal{V}$. Since $\bar{R}\left(X, V_{1}, V, W\right)=$ 0 we get by (i) that $\left[\nabla_{V} J, \nabla_{W} J\right] X$ belongs to $H$. But $\left[\nabla_{V} J, \nabla_{J W} J\right] J X=$ $-\left\{\nabla_{V} J, \nabla_{W} J\right\} X$ is equally in $H$ and the result follows.

(iv) It suffices to interchange the roles of the distributions $\mathcal{V}$ and $H$.

We can now show the following result which mainly asserts that the tangent bundle of a reducible nearly Kähler manifold contains an integrable distribution.

Proposition 3.1. Let $\left(M^{2 n}, g, J\right)$ be a complete, strict nearly Kähler manifold and let us suppose that $M$ is complex reducible and simply connected. Then $M$ splits as a Riemannian product $Z \times N$ where $Z$ and $N$ are complete and strict nearly Kähler manifolds and the tangent bundle of $N$ contains a proper $\bar{\nabla}$-parallel distribution with $\mathcal{V}$ with $\mathcal{V} \bullet \mathcal{V}=0$

Proof. We will show first that one can find a $\bar{\nabla}$-parallel distribution $\mathcal{V}$ in $T M$ such that $\mathcal{V} \bullet \mathcal{V} \subseteq \mathcal{V}$. Indeed, the reducibility of $M$ implies the existence of a $\bar{\nabla}$-parallel decomposition $T M=E \oplus F$. Let $F_{0}$ be the distribution generated by elements of the form $(v \bullet w)_{F}$ where $v, w$ belong to $E$ and the subscript denotes orthogonal projection on $F$. Using corollary 3.2 , (i) we get

$$
\left(\nabla_{X} J\right)\left(\nabla_{Y} J\right)(v \bullet w)_{E}+\left(\nabla_{X} J\right)\left(\nabla_{Y} J\right)(v \bullet w)_{F}=0
$$


whenever $X, Y$ are in $F$. But the first term of this sum belongs to $E$ by corollary 3.2, (iv) while the second is in $F$ by corollary 3.2, (ii). It follows that each term of the previous sum vanishes and this implies easily that $F \bullet F_{0}=0$. As $F_{0}$ is contained in $F$ we get that $F_{0} \bullet F_{0}=0$. Obviously, $F_{0}$ is $\bar{\nabla}$-parallel (one uses (3.3)) hence we may take $\mathcal{V}=F_{0}$ when $F_{0} \neq 0$. If $F_{0}$ then $E \bullet E \subseteq E$ and we set $\mathcal{V}=E$.

Therefore, let us consider a $\bar{\nabla}$-parallel decomposition $T M=\mathcal{V} \oplus H$, such that $\mathcal{V} \bullet \mathcal{V} \subseteq \mathcal{V}$. Let $r_{1}: V \rightarrow V$ be the tensor defined by $\left\langle r_{1} V, W\right\rangle=-\operatorname{Tr}_{\mathcal{V}}\left(\nabla_{V} J\right)\left(\nabla_{W} J\right)$ for all $V, W$ in $\mathcal{V}$. Then $r_{1}$ is parallel with respect to the unitary connection induced by $\bar{\nabla}$ in the bundle $\mathcal{V}$ thus we have the decomposition $\mathcal{V}=\mathcal{V}_{0} \oplus \mathcal{V}_{1}$ with $\mathcal{V}_{0}=\operatorname{Ker}\left(r_{1}\right)$ and $\mathcal{V}_{1}$ the orthogonal complement of $\mathcal{V}_{0}$ in $\mathcal{V}$. Since the corollary 3.2, (i) implies that $\left(\nabla_{X} J\right) r_{1} W=0$ for all $X$ in $H$ and $W$ in $\mathcal{V}$ it follows that $H \bullet \mathcal{V}_{1}=0$. Thus, we have the $\bar{\nabla}$ parallel decomposition

$$
T M=\mathcal{V}_{1} \oplus H_{1}
$$

where $H_{1}=\mathcal{V}_{0} \oplus H$. Since the very definition of $\mathcal{V}_{0}$ implies that $\mathcal{V} \bullet \mathcal{V}_{0}=0$ it is now easy to establish that $H_{1} \bullet H_{1} \subseteq H_{1}, \mathcal{V}_{1} \bullet \mathcal{V}_{1} \subseteq \mathcal{V}_{1}$ and $\mathcal{V}_{1} \bullet H_{1}=0$. It follows that the distributions $\mathcal{V}_{1}$ and $H_{1}$ are in fact $\nabla$-parallel and we conclude by the decomposition theorem of de Rham.

REMARK 3.2. In the case when $C \neq 0$ (see section 2 for definitions) proposition 3.1 can be given a simple algebraic proof. Let $\lambda_{i}, 1 \leq i \leq p$ be the (pairwise distinct) eigenvalues of $C$ ordered such that $\left|\lambda_{1}\right| \leq \ldots \leq\left|\lambda_{p}\right|$. If $E_{i}, 1 \leq i \leq p$ are the corresponding eigenspaces using the remark 2.1 it follows that $E_{p} \bullet E_{p}=0$.

Nevertheless, in the case when $C=0$ (which implies that $M$ is Einstein) the geometric arguments of the proof of the proposition 3.1 are needed.

We will show now that the class of nearly Kähler manifolds appearing in proposition 3.1 leads to another class introduced by the following definition.

DEFINITION 3.2. Let $\left(M^{2 n}, g, J\right)$ be a strict and complete nearly Kähler manifold. It is said to have special algebraic torsion if there exists a $\bar{\nabla}$-parallel decomposition $T M=\mathcal{V} \oplus H$ with $\mathcal{V} \bullet \mathcal{V}=0$ and $H \bullet H=\mathcal{V}$.

REMARK 3.3. A strict nearly Kähler manifold $(M, g, J)$ admitting a $\bar{\nabla}$-parallel decomposition $T M=\mathcal{V} \oplus H$ where $\mathcal{V} \bullet \mathcal{V}=0$ et $H \bullet H \subseteq \mathcal{V}$ is of special algebraic type. To see this, let us set $\mathcal{V}_{0}=H \bullet H$ and let $\mathcal{V}_{1}$ be the orthogonal complement of $\mathcal{V}_{0}$ in $\mathcal{V}$. By the definition of $\mathcal{V}_{1}$ we must have $\mathcal{V}_{1} \bullet H=0$ hence $\mathcal{V}_{1} \bullet T M=0$ as $\mathcal{V} \bullet \mathcal{V}=0$. The fact that $M$ is strict implies now that $\mathcal{V}_{1}=0$, thus $H \bullet H=\mathcal{V}$. Using a similar argument one can also prove that $\mathcal{V} \bullet H=H$.

Proposition 3.2. Let $\left(M^{2 n}, g, J\right)$ be a nearly Kähler manifold equipped with a $\bar{\nabla}$-parallel distribution $\mathcal{V}$ satisfaying $\mathcal{V} \bullet \mathcal{V}=0$. If $M$ is simply connected, it splits as a Riemannian product $Z \times N$ where both factors are strict nearly Kähler manifolds and $N$ has special algebraic torsion.

Proof. Set $H_{0}=\mathcal{V} \bullet H$ which is obviously contained in $H$. If $H_{1}$ is the orthogonal complement of $H_{0}$ in $H$ we get a $J$ invariant, $\bar{\nabla}$-parallel decomposition $H=H_{0} \oplus H_{1}$. Then $\mathcal{V} \bullet H_{0} \subseteq H_{0}$ and $\mathcal{V} \bullet H_{1} \subseteq H_{0}$ by the definition of $H_{0}$. But then $\mathcal{V} \bullet H_{1}$ is orthogonal to $H_{0}$ hence $\mathcal{V} \bullet H_{1}=0$. Using corollary 3.2 , (iii) we obtain that $H \bullet H_{0} \subseteq \mathcal{V}$ and since $\mathcal{V} \bullet H_{1}=0$ we must have $H_{0} \bullet H_{1}=0$. So $H_{0} \bullet H_{0} \subseteq \mathcal{V}$ and it is now easy to see that $H_{1} \bullet H_{1} \subseteq H_{1}$. Hence the decomposition $T M=E_{1} \oplus E_{2}$ is $\bar{\nabla}$-parallel with $E_{i} \bullet E_{i} \subseteq E_{i}, i=1,2, E_{1} \bullet E_{2}$ and we conclude using the de Rham decomposition theorem and the previous remark. 
The results of this section lead easily to the following theorem which roughly classifies strict nearly Kähler manifolds up to those having special algebraic torsion.

ThEOREM 3.1. Let $\left(M^{2 n}, g, J\right)$ be a complete, simply connected and strict nearly Kähler manifold. Then $M$ decomposes as Riemannian product whose factors belong to one of the following three classes of strict nearly Kähler manifolds :

-homogenous spaces of type I and II;

-Hermitian irreducible 6-dimensional manifolds;

-manifolds with special algebraic torsion.

4. Special algebraic torsion. Let $(M, g, J)$ be nearly Kähler with special algebraic torsion. Thus, we have a $\bar{\nabla}$-parallel decomposition $T M=\mathcal{V} \oplus H$ with $\mathcal{V} \bullet \mathcal{V}=0$ and $H \bullet H=\mathcal{V}$. Our first observation is that $\mathcal{V}$ being $\bar{\nabla}$-parallel with $\mathcal{V} \bullet \mathcal{V}=0$ it is integrable. We would like to establish that $\mathcal{V}$ induces on $M$ the structure of a fibration, with smooth base space. We first prove the following result, showing that the torsion tensor of the canonical Hermitian connection completely determines the curvature in the $\mathcal{V}$-direction.

Proposition 4.1. Let $X, Y$ be in $H$ and $V_{1}, V_{2}, V_{3}$ in $V$. Then :

$$
\bar{R}\left(\left(\nabla_{X} J\right) J Y, V_{1}, V_{2}, V_{3}\right)=<J Y,\left[\nabla_{V_{1}} J,\left[\nabla_{V_{2}} J, \nabla_{V_{3}} J\right]\right] X>.
$$

Moreover, we have $\bar{\nabla}_{U} \bar{R}\left(V_{1}, V_{2}, V_{3}, V_{4}\right)=0$.

Proof. Using the second Bianchi identity for the Hermitian connection $\bar{\nabla}$ (see [21]) we obtain

$$
\begin{aligned}
& \left(\bar{\nabla}_{X} \bar{R}\right)\left(Y, V_{1}, V_{2}, V_{3}\right)+\left(\bar{\nabla}_{Y} \bar{R}\right)\left(V_{1}, X, V_{2}, V_{3}\right)+\left(\bar{\nabla}_{V_{1}} \bar{R}\right)\left(X, Y, V_{2}, V_{3}\right)+ \\
& \bar{R}\left(\left(\nabla_{X} J\right) J Y, V_{1}, V_{2}, V_{3}\right)+\bar{R}\left(\left(\nabla_{Y} J\right) J V_{1}, X, V_{2}, V_{3}\right)+\bar{R}\left(\left(\nabla_{V_{1}} J\right) J X, Y, V_{2}, V_{3}\right)=0 .
\end{aligned}
$$

Since the distributions $\mathcal{V}$ and $H$ are $\bar{\nabla}$-parallel we get $\left(\bar{\nabla}_{X} \bar{R}\right)\left(Y, V_{1}, V_{2}, V_{3}\right)=$ $\left(\bar{\nabla}_{Y} \bar{R}\right)\left(V_{1}, X, V_{2}, V_{3}\right)=0$ and using lemma 3.1 and (3.3) it can be seen that the term $\left(\bar{\nabla}_{V_{1}} \bar{R}\right)\left(X, Y, V_{2}, V_{3}\right)$ also vanishes. Now, we compute the last two curvature terms in the Bianchi identity using lemma 3.1 and the corollary 3.1, (i) and the result follows by calculus. Let us prove now the second assertion. We first remark that formula (4.1) can be given the form :

$$
\left(\nabla_{X} J\right) \bar{R}\left(V_{1}, V_{2}\right) V_{3}=\left[\nabla_{V_{3}} J,\left[\nabla_{V_{1}} J, \nabla_{V_{2}}\right]\right] X
$$

Derivating this in the direction $U$ in $T M$, we get by using (3.3) that

$$
<\left(\nabla_{X} J\right)\left[\bar{\nabla}_{U} \bar{R}\left(V_{1}, V_{2}, V_{3}\right)\right], Y>=0
$$

and we conclude using the special algebraic torsion assumptions, and the strictness of $M$. D

As $H \bullet H=\mathcal{V}$ it follows that the restriction of the tensor $\bar{R}$ to the distribution $\mathcal{V}$ is completely determined by the formula (4.1). We can now prove the following.

Proposition 4.2. There exists a Riemannian manifold $(N, h)$ and a Riemannian submersion with totally geodesic fibers $\pi: M \rightarrow N$ whose vertical distribution equals $\mathcal{V}$. With respect to the induced metric and almost complex structures, each fiber is a simply connected, compact, Hermitian symmetric space. 
Proof. Consider the foliation induced by the distribution $\mathcal{V}$. One can easily show that $\mathcal{V}$ is totally geodesic hence we obtain by the second part of the proposition 4.1 that each leaf is a Hermitian symmetric space. We look now at the Ricci curvature of the leaves. Let $\widehat{R i c}: \mathcal{V} \rightarrow \mathcal{V}$ be the Ricci curvature in the vertical direction. We consider $V$ be in $\mathcal{V}$ with $V \neq 0$ and let $\left\{e_{i}\right\}$ be a local orthonormal basis in $\mathcal{V}$. Taking $V_{1}=V_{3}=e_{i}, V_{2}=V$ in (4.2), we obtain after taking the trace over $H$ that :

$$
<\widehat{\operatorname{Ric}}(V), r(V)>=2 \sum_{e_{i} \in \mathcal{V}}\left\|\left(\nabla_{V} J\right)\left(\nabla_{e_{i}} J\right)\right\|^{2}>0
$$

(here, we considered that the norm of the linear operator $A$ on $T M$ is given by $\left.\|A\|^{2}=\operatorname{Tr}\left(A A^{\star}\right)\right)$. Now the tensor $r$ preserves $\mathcal{V}$ and its restriction to $\mathcal{V}$ is $\nabla$-parallel with strictly positive eigenvalues. It follows then easily that each leaf has positive Ricci curvature and we use a result of [20] to obtain that the leaves are compact and simply connected. The simple connectivity of the leaves implies that the foliation has trivial leaf holonomy, so using the criterion from [34], page 90, we get a smooth fibration $\pi: M \rightarrow N$. The metric part of our statement is a standard one, which is left to the reader.

We will refer to the fibration $\pi: M \rightarrow N$ as the canonical fibration of the nearly Kähler manifold with special algebraic torsion $M$. Its generic fiber will be denoted by $F$. Before going further, let us examine a few geometric properties related to special algebraic torsion.

Proposition 4.3. Let $(M, g, J)$ be a nearly Kähler with special algebraic torsion and corresponding decomposition $T M=\mathcal{V} \oplus H$. Define a Riemannian metric $\bar{g}$ on $M$ by $\bar{g}=2 g_{\mathcal{V}} \oplus g_{H}$ where $g_{\mathcal{V}}$ and $g_{H}$ are the restrictions of the metric $g$ to the distributions $\mathcal{V}$ and $H$ respectively. We also define a new almost complex structure $\bar{J}$ by setting $\bar{J}_{\mathcal{V}}=-J$ and $\bar{J}_{\mid H}=J$. Then $(M, \bar{g}, \bar{J})$ is a Kähler manifold of positive Ricci curvature, and hence simply connected.

Proof. The proof of the fact that $(M, \bar{g}, \bar{J})$ is Kähler is essentially the same with the one given in the 6-dimensional case in [1]. Let us denote by $\tilde{\nabla}$ the Levi-Civita connection of the metric $\bar{g}$. In the standard way we get

$$
\begin{aligned}
& \widetilde{\nabla}_{X} Y=\nabla_{X} Y, \widetilde{\nabla}_{X} V=\bar{\nabla}_{X} V-\frac{1}{2}\left(\nabla_{X} J\right) J V \\
& \widetilde{\nabla}_{V} X=\bar{\nabla}_{V} X, \widetilde{\nabla}_{V} W=\nabla_{V} W
\end{aligned}
$$

whenever $X, Y$ are in $H$ and $V, W$ in $\mathcal{V}$. After a routine computation we obtain

$$
\begin{aligned}
& R i c_{\tilde{g}}=R i c_{g}-\frac{r}{4} \text { on } H \\
& R i c_{\tilde{g}}=R i c_{g}+\frac{3 r}{4} \text { on } \mathcal{V}
\end{aligned}
$$

and of course $\operatorname{Ric}_{\bar{g}}(V, X)=0$ for $V, X$ belonging to $\mathcal{V}$ and $H$ respectively. Using (2.3) we see that the Ricci tensor of $\tilde{g}$ is strictly positive and by a theorem of Kobayashi [20] $M$ is simply connected.

We are now going to prove that the standard de Rham decomposition of the fiber induces a splitting at the level of the canonical fibration.

THEOREM 4.1. Let $(M, g, J)$ be a nearly Kähler manifold with special algebraic torsion. Then $M$ is a Riemannian product

$$
M_{1} \times M_{2} \times \ldots \times M_{q}
$$


where each $M_{i}, 1 \leq i \leq q$, is a nearly Kähler manifold with special algebraic torsion and such that the canonical fibration has irreducible fiber.

Proof. If the fiber $F$ is irreducible there is nothing to prove. Otherwise it decomposes as $F_{1} \times F_{2}$ and using parallel transport we obtain a $\nabla$-parallel decomposition $\mathcal{V}=\mathcal{V}^{1} \oplus \mathcal{V}^{2}$. Hence $R\left(\left(\nabla_{X} J\right) Y, V, V_{1}, V_{2}\right)=0$ for all $X, Y$ in $H, V$ in $\mathcal{V}$ and $V_{i}$ in $\mathcal{V}^{i}, i=1,2$. Using (4.1) we obtain

$$
\left[\nabla_{V} J,\left[\nabla_{V_{1}} J, \nabla_{V_{2}} J\right]\right]=0
$$

Taking $V=V_{1}$ we get :

$$
\left(\nabla_{V_{1}} J\right)^{2}\left(\nabla_{V_{2}} J\right)+\left(\nabla_{V_{2}} J\right)\left(\nabla_{V_{1}} J\right)^{2}=2\left(\nabla_{V_{1}} J\right)\left(\nabla_{V_{2}} J\right)\left(\nabla_{V_{1}} J\right)
$$

We change $V_{2}$ in $J V_{2}$ in the previous relation and we use that $\left(\nabla_{J V_{2}} J\right)=\left(\nabla_{V_{2}} J\right) J$. We get

$$
\left.\left(\nabla_{V_{1}} J\right)^{2}\left(\nabla_{V_{2}} J\right)+\left(\nabla_{V_{2}} J\right)\left(\nabla_{V_{1}} J\right)^{2}=-2\left(\nabla_{V_{1}} J\right) \nabla_{V_{2}} J\right)\left(\nabla_{V_{1}} J\right)
$$

hence we must have

$$
\left(\nabla_{V_{1}} J\right)^{2}\left(\nabla_{V_{2}} J\right)+\left(\nabla_{V_{2}} J\right)\left(\nabla_{V_{1}} J\right)^{2}=\left(\nabla_{V_{1}} J\right)\left(\nabla_{V_{2}} J\right)\left(\nabla_{V_{1}} J\right)=0
$$

This implies that $\left(\nabla_{V_{1}} J\right)^{3}\left(\nabla_{V_{2}}\right)=0$ and since $-\left(\nabla_{V_{1}} J\right)^{2}$ is a nonnegative operator we have that $\left(\nabla_{V_{1}} J\right)^{2}\left(\nabla_{V_{2}} J\right)=0$. We equally have $\left(\nabla_{V_{2}} J\right)\left(\nabla_{V_{1}} J\right)^{2}=0$. It follows that

$$
\left\|\left(\left(\nabla_{V_{2}} J\right)\left(\nabla_{V_{1}} J\right)\right) X\right\|^{2}=\left\|\left(\left(\nabla_{V_{1}} J\right)\left(\nabla_{V_{2}} J\right)\right) X\right\|^{2}=0
$$

for all $X$ in $H$, that is

$$
\left.\left.\left(\nabla_{V_{2}} J\right)\left(\nabla_{V_{1}} J\right)\right)=\left(\nabla_{V_{1}} J\right)\left(\nabla_{V_{2}} J\right)\right)=0
$$

whenever $V_{1}$ and $V_{2}$ belong to $\mathcal{V}_{1}$ and $\mathcal{V}_{2}$ respectively. We define now the subspaces $H^{1}$ and $H^{2}$ of $H$ by

$$
H^{i}=\mathcal{V}^{i} \bullet H, i=1,2
$$

Using (4.4) and the fact that $\mathcal{V} \bullet H=H$ (see remark 3.3) we easily obtain that $H=H^{1} \oplus H^{2}$. After standard manipulations it follows that $\mathcal{V}^{1} \bullet H^{2}=\mathcal{V}^{2} \bullet H^{1}=$ $H^{1} \bullet H^{2}=0$ and further that $\mathcal{V}^{i} \bullet H^{i}=H^{i}, H^{i} \bullet H^{i}=\mathcal{V}^{i}$ for $i=1,2$. We are going to show now that the four distributions $\mathcal{V}^{1}, \mathcal{V}^{2}, H^{1}, H^{2}$ are $\bar{\nabla}$-parallel. Using that $\mathcal{V}^{1} \bullet H^{1}=H^{1}$ and (3.3) we establish that $H^{1}$ is $\bar{\nabla}$-parallel. For the parallelism of $\mathcal{V}^{1}$ we use that $H^{1} \bullet H^{1}=\mathcal{V}^{1}$ and the remaining cases are analogous. Now, the distributions $E_{i}=\mathcal{V}^{i} \oplus H^{i}, i=1,2$ are $\bar{\nabla}$-parallel. Since $E_{i} \bullet E_{i} \subseteq E_{i}, i=1,2$ and $E_{1} \bullet E_{2}=0$ they are in fact $\nabla$-parallel and we conclude by the de Rham's theorem, as $M$ is simply connected. $\square$

We can therefore admit without loss of generality that the fiber $F$ of the canonical fibration is an irreducible Riemannian manifold. This assumption is to be made in the rest of the present section.

Let us define a tensor $\mathcal{R}: H \times H \rightarrow$ End_ $_{-}(H)$ by

$$
\mathcal{R}(X, Y)=\bar{R}(X, Y)+\nabla_{\left(\nabla_{X} J\right) Y}
$$


for all $X, Y$ in $H$. Now, if $X^{\star}$ denotes the horizontal lift of the vector field $X$ of $N$, using standard computations from [2], page 241, we get

$$
\mathcal{R}\left(X^{\star}, Y^{\star}\right) Z^{\star}=\left(R^{h}(X, Y) Z\right)^{\star}
$$

whenever $X, Y, Z$ are vector fields on $N$ (here $R^{h}$ denotes the curvature tensor of $N$ ).

LEMMA 4.1. We have

$$
\sum_{e_{i} \in H} \mathcal{R}\left(e_{i},\left(\nabla_{V} J\right) e_{i}\right)=\nabla_{r(V)} J
$$

for all $V$ in $\mathcal{V}$ and all orthonormal basis $\left\{e_{i}\right\}$ of $H$.

Proof. We compute the given sum using the base $\left\{J e_{i}\right\}$ and we take into account that $\bar{R}(J X, J Y)=\bar{R}(X, Y)$ whenever $X, Y$ belong to $H$. $\square$

THEOREM 4.2. The Riemannian manifold $(N, h)$ is irreducible.

Proof. If $(M, h)$ wasn't irreducible its tangent bundle admitted a decomposition $T N=E_{1} \oplus E_{2}$ parallel with respect of the Levi-Civita connection of $h$, to be denoted by $\nabla^{h}$. We obviously have $H=H_{1} \oplus H_{2}$ where

$$
H_{i}=\left\{v \in H:(d \pi) v \in E_{i}\right\}
$$

for $i=1,2$. Now, the operator $R^{h}(X, Y)$ preserves $E_{1}$ and $E_{2}$ for all $X, Y$ in $T N$. It follows by (4.5) that $\mathcal{R}(X, Y)$ preserves $H_{1}$ and $H_{2}$ whenever $X$ and $Y$ are in $H$. Hence lemma 4.1 easily implies that $\mathcal{V} \bullet H_{i} \subseteq H_{i}$ for $i=1,2$. It follows that $H_{1} \bullet H_{2}$ and $\mathcal{V}$ are orthogonal but the fact that $H_{1} \bullet H_{2} \subseteq \mathcal{V}$ gives that $H_{1} \bullet H_{2}=0$. We get a decomposition

$$
T M=\mathcal{V}^{\prime} \oplus H_{2}
$$

where $\mathcal{V}^{\prime}=\mathcal{V}^{\prime} \oplus H_{1}$. This decomposition satisfies $\mathcal{V}^{\prime} \bullet \mathcal{V}^{\prime} \subseteq \mathcal{V}^{\prime}$ and $H_{2} \bullet H_{2} \subseteq \mathcal{V}^{\prime}$. Let us show now that it is also $\bar{\nabla}$-parallel.

As $\bar{\nabla}_{X^{\star}} Y^{\star}=\left(\nabla_{X} Y\right)^{\star}$ for all $X, Y$ in $T N$ it follows that $\bar{\nabla}_{X} Y$ belongs to $H_{i}$ whenever $X$ is in $H$ and $Y$ is in $H_{i}, i=1,2$. It remains to see that $\bar{\nabla}_{V} X$ belongs to $H_{i}$ whenever $X$ is in $H_{i}, V$ is in $\mathcal{V}$ and $i=1,2$. Indeed if $X$ is in $E_{1}$ we know that $\left[V, X^{\star}\right]$ is in $\mathcal{V}$ so by the parallelism of $H$ is follows that $\bar{\nabla}_{V} X^{\star}=-\left(\nabla_{V} J\right) J X^{\star}$ which belongs to $H_{1}$ as we already shown that $\mathcal{V} \bullet H_{1} \subseteq H_{1}$. Thus, $H_{1}$ is $\bar{\nabla}$-parallel and of course the same holds for $\mathrm{H}_{2}$.

Hence we may apply corollary 3.2, (i) for the distribution $\mathcal{V}^{\prime}$ and we get

$$
\left(\nabla_{X} J\right)\left(\nabla_{V} J\right) W=0
$$

for $V, W$ in $\mathcal{V}^{\prime}$ and $X$ in $H_{2}$. If we set $\mathcal{V}^{i}=H_{i} \bullet H_{i}, i=1,2$ it follows that $\mathcal{V}^{1} \bullet H_{2}=0$. Applying the same reasoning to the decomposition $T M=\mathcal{V}^{\prime \prime} \oplus H_{1}$ where $\mathcal{V}^{\prime \prime}=\mathcal{V} \oplus H_{1}$ we get $\mathcal{V}^{2} \bullet H_{1}=0$. It is now routine to verify that $\mathcal{V}^{1}$ and $\mathcal{V}^{2}$ are orthogonal thus $\mathcal{V}=\mathcal{V}^{1} \oplus \mathcal{V}^{2}$. Since $\mathcal{V}^{i}, i=1,2$ are $\nabla$-parallel (one uses the formula 3.3) by the irreducibility of $\mathcal{V}$ we must have, say $\mathcal{V}^{1}=0$. But this would imply $H_{1} \bullet \mathcal{V}=0$ and further $H_{1} \bullet T M=0$ a contradiction, since $(M, g, J)$ is strict.

To summarize the results of this section, nearly Kähler manifolds with special algebraic torsion have the property that up to Riemannian products of the total space, the fiber (a compact, simply connected, Hermitian symmetric space) and the base space of the canonical fibration are irreducible Riemannian manifolds. 
5. Metric properties. In this section we will consider $\left(M^{2 n}, g, J\right)$, a nearly Kähler manifold with special algebraic torsion and decomposition $T M=\mathcal{V} \oplus H$. We also suppose that the fiber of the canonical fibration is irreducible and recall that the base space has to be irreducible, too. Let us define a tensor $F: H \rightarrow H$ by

$$
F=-\sum_{e_{i} \in \mathcal{V}}\left(\nabla_{e_{i}} J\right)^{2}
$$

where $\left\{e_{i}\right\}$ is a local orthonormal frame in $\mathcal{V}$. Our main objective will be to determine the maximal number of eigenvalues of the tensors $F$ and $C$. This will be used for the final classification result in the next section. It will also separate the nearly Kähler manifolds with special algebraic torsion into two classes.

The fact that $C$ is $\bar{\nabla}$-parallel and symmetric, together with the irreducibilty of the fiber imply that there exists a real constant $\lambda$ such that $C=\lambda 1_{\mathcal{V}}$ on $\mathcal{V}$. This leads to the following:

Proposition 5.1. The tensor $C$ has at most three eigenvalues.

Proof. We know that $-C(V \bullet X)=V \bullet C X+C V \bullet X$ for all $V$ in $\mathcal{V}$ and $X$ in $H$ (see section 2). As $\mathcal{V}$ is an eigenspace of $C$ with eigenvalue $\lambda$ this implies that $S(V \bullet X)+V \bullet S X=0$ whenever $V, X$ are in $\mathcal{V}$ and $H$ respectively, where $S=C+\frac{\lambda}{2}$ on $H$. Let us denote by $\mathcal{L}_{V}^{H}$ the projection of the Lie derivative $\mathcal{L}_{V}$ on $H$. Taking into account that $S^{2}$ is $\bar{\nabla}$-parallel it follows using the above algebraic property of $S$ that $\mathcal{L}_{V}^{H} S^{2}=0$ for all $V$ in $\mathcal{V}$. It is now an elementary exercise to see that $S^{2}$ projects on a symmetric, $\nabla^{h}$-parallel endomorphism of $N$ which has to be a multiple of identity by the irreducibility of $N$, hence proving our assertion.

Using the fact that $H \bullet H=\mathcal{V}$ it is straightforward to show that $r_{\mid H}=2 F$. Hence when $C$ has a single eigenvalue, that is $C=0$ it follows also that $F$ has a single eigenvalue. We concentrate now on the cases when $C$ has two or three eigenvalues. We will need several preliminary results.

LEMMA 5.1. We have :

$$
\operatorname{Ric}\left(\left(\nabla_{X} J\right) Y, V\right)=\frac{1}{4} r\left(V,\left(\nabla_{X} J\right) Y\right)+<Y, F(V \bullet X)+V \bullet F X>
$$

whenever $X, Y$ are in $H$ and $V$ is in $\mathcal{V}$.

(ii) The base manifold $(N, h)$ is Einstein with Einstein constant $\mu>0$ and furthermore

$$
\operatorname{Ric}+\frac{r}{4}=\mu \cdot 1_{H}
$$

on $H$.

Proof. (i) is an immediate consequence of (4.2). To prove (ii), let $X, Y$ be vector fields on $N$. Then $\operatorname{Ric}^{h}(X, Y) \circ \pi=\sum_{e_{i} \in H} \mathcal{R}\left(X^{\star}, e_{i}, Y^{\star}, e_{i}\right)=\frac{1}{2}<r\left(X^{\star}\right), Y^{\star}>$ $+\operatorname{Ric}_{\bar{\nabla}}\left(X^{\star}, Y^{\star}\right)$ where $\left\{e_{i}\right\}$ is a local orthonormal frame in $H$. But $\operatorname{Ric}_{\bar{\nabla}}=\frac{1}{4}(3 R i c+$ $\operatorname{Ric}^{\star}$ ) (see [14]) thus

$$
\operatorname{Ric}^{h}(X, Y) \circ \pi=<\left(R i c+\frac{r}{4}\right) X^{\star}, Y^{\star}>
$$

Recall that $r$ is a $\bar{\nabla}$-parallel tensor, as well as Ric (see [24]). Hence, it is straightforward to see that $\operatorname{Ric}^{h}$ is $\nabla^{h}$-parallel and since $(N, h)$ is irreducible, we must have 
$\operatorname{Ric}^{h}=\mu \cdot h$ for some constant $\mu$. That $\mu>0$ follows by the fact that the Ricci curvature of $g$ is strictly positive (see [24]).

We mention now without proof the following elementary result.

LEMMA 5.2. Let $Q$ be a $\bar{\nabla}$-parallel subbundle of $H$ such that $\mathcal{V} \bullet Q=0$. Then there exists a $\nabla^{h}$-parallel subbundle $E$ of $T N$ such that $Q=\pi^{\star} E$.

We are now able to treat the cases when the tensor $C$ admits exactly two or three eigenvalues.

Proposition 5.2. (i) If the tensor $C$ has exactly two eigenvalues then $F=k \cdot 1_{H}$ with $k>0$ constant. Furthermore, the eigenvalues and eigenbundles of each of the tensors $r, C$, Ric are given in the following table.

\begin{tabular}{|r|c|c|c|c|}
\hline Eigenvalue & $r$ & Ric & $C$ & Eigenbundle \\
\hline$\lambda_{1}$ & $\frac{n-d}{d} k$ & $\frac{n+7 d}{4 d} k$ & $\frac{4(n-3 d)}{d} k$ & $\mathcal{V}$ \\
\hline$\lambda_{2}$ & $2 k$ & $\frac{n+2 d}{2 d} k$ & $-\frac{2(n-3 d)}{d} k$ & $H$ \\
\hline
\end{tabular}

Here $d$ is the half dimension of the distribution $\mathcal{V}$.

(ii) Suppose that the tensor $C$ has exactly three eigenvalues. Then the tangent bundle of $M$ admits a $\bar{\nabla}$-parallel decomposition

$$
T M=\mathcal{V} \oplus H_{1} \oplus H_{2}
$$

with $H_{1} \bullet H_{1}=H_{2} \bullet H_{2}=0$ and $H_{1} \bullet H_{2}=\mathcal{V}$. Moreover, $(M, g)$ is a homogenous space and the base space of the canonical fibration is a simply connected, compact and irreducible symmetric space.

Proof. (i) Using the (2.2) it easy to see that the eigenvalues of $C$ are of the form $\lambda$ and $-\frac{\lambda}{2}$. Since by definition $C=R i c-5 R i c^{\star}$ we get by $(5.2)$ that $r=\frac{-\lambda+8 \mu}{12}$ on $H$ hence $F$ has a single eigenvalue.

(ii) Let $\lambda_{1}$ and $\lambda_{2}$ be the eigenvalues of $C$ on $H$, and denote the corresponding eigenbundles by $H_{1}$ and $H_{2}$. Using lemma 5.2 we have that $\mathcal{V} \bullet H_{1} \neq 0$, hence it follows by (2.2) that $-\left(\lambda+\lambda_{1}\right)$ is an eigenvalue of $C$. It is different from $\lambda$ since $\mathcal{V} \bullet H_{1}$ is orthogonal to $\mathcal{V}$. Let us suppose that $-\left(\lambda+\lambda_{1}\right)=\lambda_{1}$. It follows that $\mathcal{V} \bullet H_{1} \subseteq H_{1}$ and since $M$ has special algebraic torsion we get that $H_{2} \bullet \mathcal{V}$ is orthogonal ot $\mathcal{V} \oplus H_{1}$, that is $H_{2} \bullet \mathcal{V}$ is contained in $H_{2}$. Again by lemma 5.2 we have $H_{2} \bullet \mathcal{V} \neq 0$ and using (2.2) we find that $\lambda_{2}=-\frac{\lambda}{2}=\lambda_{1}$ an absurdity.

Hence $-\left(\lambda+\lambda_{1}\right)=\lambda_{2}$ a fact which obviously implies by (2.2) that $\mathcal{V} \bullet H_{1} \subseteq$ $H_{2}, \mathcal{V} \bullet H_{2} \subseteq H_{1}$ and $H_{1} \bullet H_{2} \subseteq \mathcal{V}$. Let us show now that $H_{1} \bullet H_{1}=0$. If $H_{1} \bullet H_{1} \neq 0$ it produces the eigenvalue $-2 \lambda_{2}$ for $C$. Or $H_{1} \bullet H_{1}$ is orthogonal to $H_{2}$ and $-2 \lambda_{1} \neq \lambda$ (since $\lambda_{1} \neq \lambda_{2}$ ). We get $-2 \lambda_{1}=\lambda_{1}$ hence $\lambda_{1}=0$ and $H_{1} \bullet H_{1} \subseteq H_{1}$. We set $\mathcal{V}^{\prime}=H_{1}$ and $H^{\prime}=\mathcal{V} \oplus H_{2}$. Then using corollary 3.2 , (ii) we obtain that $\left(\nabla_{X} J\right)\left(\nabla_{Y} J\right) Z$ belongs to $H^{\prime}$ whenever $X, Y$ are in $\mathcal{V}$ and $Z$ is in $H_{1} \bullet H_{1}$. As $\mathcal{V} \bullet\left(\mathcal{V} \bullet H_{1}\right) \subseteq \mathcal{V} \bullet H_{2} \subseteq H_{1}$ we obtain easily that $\mathcal{V} \bullet\left(H_{1} \bullet H_{1}\right)=0$ and we conclude by lemma 5.2 . In the same way it can be proven that $\mathrm{H}_{2} \bullet \mathrm{H}_{2}=0$. Hence, we have three different splittings of special algebraic type $T M=\mathcal{V} \oplus H, T M=H_{1} \oplus\left(\mathcal{V} \oplus H_{2}\right), T M=H_{2} \oplus\left(\mathcal{V} \oplus H_{1}\right)$ each of which being $\bar{\nabla}$-parallel. It follows that the restriction of the tensor $\bar{\nabla}_{U} \bar{R}$ to either $\mathcal{V}, H_{1}$ or $H_{2}$ vanishes. Or this implies easily that $\bar{\nabla} \bar{R}=0$, so $\bar{\nabla}$ is an Ambrose-Singer connection hence $(M, g, J)$ is a homogenous space [28]. That the base space of the canonical fibration is symmetric follows by the usual comparison between curvature tensors of the total and the base space in [2]. $\square$ 
Hence we obtained a new class of nearly Kähler manifolds motivating the following definition.

Definition 5.1. A nearly Kähler manifold is homogenous of type III if it has special algebraic torsion and the tensor $C$ admits exactly three eigenvalues.

REMARK 5.1. In the case when $C$ has exactly three eigenvalues, let $d_{1}$ and $d_{2}$ be the half dimensions of $H_{1}$ and $H_{2}$ respectively. Then the eigenvalues of the tensors $r, R i c, C$ with their corresponding eigenbundles are listed in the following table.

\begin{tabular}{|r|c|c|c|c|}
\hline Eigenvalue & $r$ & Ric & $C$ & Eigenbundle \\
\hline$\lambda_{1}$ & $\frac{2 d_{1}}{d} k$ & $\left(\frac{d_{1}}{2 d}+\frac{d_{1}}{d_{2}}+1\right) k$ & $4\left(\frac{2 d_{1}}{d}-\frac{d_{1}}{d_{2}}-1\right)$ & $\mathcal{V}$ \\
\hline$\lambda_{2}$ & $k$ & $\left(\frac{d_{1}}{d}+\frac{d_{1}}{d_{2}}+\frac{1}{2}\right) k$ & $-4\left(\frac{d_{1}}{d}+\frac{d_{1}}{d_{2}}-2\right)$ & $H_{1}$ \\
\hline$\lambda_{3}$ & $\frac{d_{1}}{d_{2}} k$ & $\left(\frac{d_{1}}{d}+\frac{d_{1}}{2 d_{2}}+1\right) k$ & $-4\left(\frac{d_{1}}{d}-\frac{2 d_{1}}{d_{2}}+1\right)$ & $H_{2}$ \\
\hline
\end{tabular}

Here, $k$ is a positive constant and, moreover, the eigenvalues of the tensor $F$ are $k$ and $\frac{d_{1}}{d_{2}} k$ with eigenbundles $H_{1}$ and $H_{2}$ respectively. The proof will be omitted, as being a simple calculation, based on (2.3) and of the relations between eigenvalues of the tensors $C, F$ and $r$ developed in the proof of the proposition 5.2, (ii).

To summarize the results obtained so far in this section, one can reduce the study of special algebraic torsion to the case when the tensor $F$ has a single eigenvalue.

6. The holonomy of the base manifold. We consider in this section a nearly Kähler manifold with special algebraic torsion and let $T M=\mathcal{V} \oplus H$ be the corresponding decomposition. Furthermore, we suppose that the fiber of the canonical fibration is irreducible and that the tensor $F$ has a single eigenvalue, to be denoted by $k$.

Let $m$ be a point of $M$. We define a vector subspace of $\mathfrak{s o}\left(H_{m}\right)$ by

$$
\mathfrak{p}_{m}=\left\{\nabla_{v} J: v \text { in } \mathcal{V}_{m}\right\}
$$

The dimension of $\mathfrak{p}_{m}$ equals that of $\mathcal{V}_{m}$ since $(M, g, J)$ is strict. Let $\mathfrak{k}_{m}$ be the vector subspace of $\mathfrak{s o}\left(H_{m}\right)$ generated by

$$
\left\{\left[\nabla_{v} J, \nabla_{w} J\right]: v, w \in \mathcal{V}_{m}\right\}
$$

Proposition 6.1. We have $\mathfrak{k}_{m} \cap \mathfrak{p}_{m}=0$. Then $\mathfrak{h}_{m}=\mathfrak{k}_{m} \oplus \mathfrak{p}_{m}$ is a Lie subalgebra of $\mathfrak{s o}\left(H_{m}\right)$.

Proof. Let us first prove that $\left[\mathfrak{p}_{m}, \mathfrak{k}_{m}\right] \subseteq \mathfrak{p}_{m}$. Indeed, if $V_{1}, V_{2}, V_{3}$ belong to $\mathcal{V}$ we obtain using (4.2) that

$$
\left[\nabla_{V_{1}} J,\left[\nabla_{V_{2}} J, \nabla_{V_{3}} J\right]=-\nabla_{R\left(V_{2}, V_{3}\right) V_{1}} J\right.
$$

and the assertion is proven. Consider now $z$ in $\mathfrak{p}_{m} \cap \mathfrak{k}_{m}$. Then $z=\nabla_{v} J$ with $v$ in $\mathcal{V}$ and $\left[\nabla_{v} J, x\right]$ belongs to $\mathfrak{p}_{m}$ for all $x$ in $\mathfrak{p}$. In particular, there exists $w$ in $\mathcal{V}$ such that $\left[\nabla_{v} J, \nabla_{J v} J\right]=\nabla_{w} J$, which implies $2\left(\nabla_{v} J\right)^{2}=\nabla_{J w} J$. The left side of this equality is symmetric whilst the right is skew-symmetric. It follows that $z=0$ and we proved that $\mathfrak{k}_{m} \cap \mathfrak{p}_{m}=0$. Now, $\left[\mathfrak{p}_{m}, \mathfrak{p}_{m}\right] \subseteq \mathfrak{k}_{m}$ by definition and using the Jacobi identity and (4.2) we obtain

$$
\left[\left[\nabla_{V_{1}} J, \nabla_{V_{2}} J\right],\left[\nabla_{V_{3}} J, \nabla_{V_{4}} J\right]\right]=\left[\nabla_{V_{2}} J, \nabla_{R\left(V_{3}, V_{4}\right) V_{1}} J\right]+\left[\nabla_{R\left(V_{3}, V_{4}\right) V_{2}} J, \nabla_{V_{1}} J\right]
$$


for all $V_{i}$ in $\mathcal{V}, 1 \leq i \leq 4$. It follows that $\mathfrak{k}_{m}$ and $\mathfrak{h}_{m}$ are Lie algebras.

REMARK 6.1. (i) We saw along the proof of the previous proposition that $\left[\mathfrak{p}_{m}, \mathfrak{p}_{m}\right] \subseteq \mathfrak{k}_{m}$ and $\left[\mathfrak{p}_{m}, \mathfrak{k}_{m}\right] \subseteq \mathfrak{p}_{m}$.

(ii) Using E. Cartan's description of symmetric spaces one can also relate the Lie algebras $\mathfrak{h}_{m}, \mathfrak{k}_{m}$ to the homogenenous structure of the symmetric space $F_{m}$, the fiber of $\pi$ through $\pi(m)$.

We will now relate the Lie algebra $\mathfrak{h}_{m}$ to the holonomy group of the base manifold. We define the Lie algebra $\mathfrak{h o l}_{m}$ as the Lie subalgebra of $\mathfrak{s o}\left(H_{m}\right)$ generated by skewsymmetric endomorphisms of the form

$$
\tau_{c}^{-1} \circ \mathcal{R}\left(\tau_{c} v, \tau_{c} w\right) \circ \tau
$$

where $v, w$ belong to $H_{m}, c$ is a horizontal curve in $M$ starting at $m$ and $\tau_{c}$ is parallel transport along $c$ with respect to the connection $\bar{\nabla}$. Using standard considerations in holonomy theory we find that $\mathfrak{h o l}_{m}$ is in fact isomorphic with the Lie algebra of the holonomy group of $(N, h)$ through $\pi m$.

LEMMA 6.1. The Lie algebra $\mathfrak{h}_{m}$ is an ideal of $\mathfrak{h o l}_{m}$.

Proof. Let us first note that (3.3) gives us, after some computations

$$
\left[\bar{R}(X, Y), \nabla_{V} J\right]=\nabla_{\bar{R}(X, Y) V} J
$$

for all $X, Y$ in $H$ and $V$ in $\mathcal{V}$. It follows that $\left[\mathcal{R}(v, w), \mathfrak{p}_{m}\right] \subseteq \mathfrak{h}_{m}$ and since $\left[\mathfrak{p}_{m}, \mathfrak{p}_{m}\right]=$ $\mathfrak{k}_{m}$ by the Jacobi identity we also get $\left[\mathcal{R}(v, w), \mathfrak{k}_{m}\right] \subseteq \mathfrak{h}_{m}$ for all $v, w$ in $H_{m}$. Let $c$ be a horizontal path in $M$ starting at $m$. Using (3.3) we obtain easily that $\tau_{c} \circ \nabla_{v} J=$ $\left(\nabla_{\tau_{c} v} J\right) \circ \tau_{c}$ whenever $v$ belongs to $H_{m}$. The conclusion now follows. $\square$

Let us consider now the bundle $\mathfrak{h}$ whose fiber at a point $m$ of $M$ equals $\mathfrak{h}_{m}$. Note that $\mathfrak{h}$ can be identified, in a standard way, with a subbundle of $\Lambda^{2}(H)$. We do this identification tacitly in the rest of this section. In the same way we obtain bundles $\mathfrak{k}, \mathfrak{p}$. We need now the following metric fact.

Proposition 6.2. We have $\rho^{\mathcal{R}}=\frac{k(n-d)}{d}$ on $\mathfrak{h}$ where we denoted by $\rho^{\mathcal{R}}$ the curvature operator of $\mathcal{R}$.

Proof. That the claimed formula holds on $\mathfrak{p}$ was proved in lemma 4.2 (see also table 5.3). Let us show that it holds on $\mathfrak{k}$. We must compute $\sum_{e_{i} \in H} \mathcal{R}\left(e_{i}, q e_{i}, X, Y\right)$ where $e_{i}$ is a local orthonormal basis in $H$ and $q=\left[\nabla_{V} J, \nabla_{W} J\right]$ with $V, W$ in $\mathcal{V}$. The definition of $C$ implies easily that our quantity equals $\sum_{e_{i} \in H} \bar{R}\left(e_{i}, q e_{i}, X, Y\right)+\sum_{e_{i} \in H} \nabla_{\left(\nabla_{e_{i}} J\right) q e_{i}} J$. Or $\sum_{e_{i} \in H}\left(\nabla_{e_{i}} J\right) q e_{i}=0$ (one uses the base $J e_{i}$ ). Now, using the first Bianchi identity for the Hermitian connection $\bar{\nabla}$ (see [21]) we get :

$$
\begin{aligned}
& \sum_{e_{i} \in H} \bar{R}\left(e_{i}, q e_{i}, X, Y\right)+\sum_{e_{i} \in H} \bar{R}\left(q e_{i}, X, e_{i}, Y\right)+\sum_{e_{i} \in H} \bar{R}\left(X, e_{i}, q e_{i}, Y\right)= \\
& =\operatorname{Tr}_{H}\left[\nabla_{Y} J, \nabla_{X} J\right] q .
\end{aligned}
$$

But $\sum_{e_{i} \in H} \bar{R}\left(q e_{i}, X, e_{i}, Y\right)=\sum_{e_{i} \in H}<\left[\bar{R}\left(X, e_{i}\right), q\right] e_{i}, Y>+\operatorname{Ric}_{\bar{\nabla}}(X, q Y)$ where $\operatorname{Ric}_{\bar{\nabla}}$ denotes the Ricci tensor of the Hermitian connection $\bar{\nabla}$. The use of (6.1) implies that $<\left[\bar{R}\left(X, e_{i}\right), q\right] e_{i}, Y>=<\left[\nabla_{V} J, \nabla_{\bar{R}\left(X, e_{i}\right) W} J\right] e_{i}, Y>-<\left[\nabla_{W} J, \nabla_{\bar{R}\left(X, e_{i}\right) V} J\right] e_{i}, Y>$. 
Now using lemma 3.1 we obtain after computing at some length that

$$
\begin{aligned}
& \left.\sum_{e_{i} \in H}<\left[\nabla_{V} J, \nabla_{\bar{R}\left(X, e_{i}\right) W} J\right] e_{i}, Y>=-<\left(\nabla_{W} J\right) X, G\left(\nabla_{V} J\right) Y\right)>- \\
& -\operatorname{Tr}_{H}\left(\nabla_{X} J\right)\left(\nabla_{Y} J\right)\left(\nabla_{V} J\right)\left(\nabla_{W} J\right) .
\end{aligned}
$$

where $G: H \rightarrow H$ is defined by $G=-\sum_{e_{i} \in H}\left(\nabla_{e_{i}} J\right)^{2}$. It follows that

$$
\begin{aligned}
& \sum_{e_{i} \in H}<\left[\bar{R}\left(X, e_{i}\right), q\right] e_{i}, Y>=-\operatorname{Tr}_{H}\left(\nabla_{X} J\right)\left(\nabla_{Y} J\right) q+<\left(\nabla_{V} J\right) X, G\left(\nabla_{W} J\right) Y>- \\
& <\left(\nabla_{W} J\right) X, G\left(\nabla_{V} J\right) Y>.
\end{aligned}
$$

Since $G=k 1_{H}$ we finally obtain that

$$
\sum_{e_{i} \in H} \bar{R}\left(e_{i}, q e_{i}, X, Y\right)+\operatorname{Ric}_{\bar{\nabla}}(X, q Y)-\operatorname{Ric}_{\bar{\nabla}}(Y, q X)+2 k<q X, Y>=0
$$

We conclude by the fact (see [14]) that $\operatorname{Ric}_{\bar{\nabla}}=\frac{1}{4}\left(3 R i c+R i c^{\star}\right)$ on $M$ and by using the proposition 5.2 , (i). $\square$

We are now ready to prove :

Theorem 6.1. Let $(M, g, J)$ be a nearly Kähler manifold with special algebraic torsion and let us suppose that the fiber of the canonical fibration is irreducible. If the tensor $F$ has a single eigenvalue and the base space of the canonical fibration is not symmetric then $M$ is the twistor space over a quaternionic-Kähler manifold of positive scalar curvature.

Proof. The base space $\left(N^{2 m}, h\right)$ of the canonical fibration is an irreducible, Einstein manifold of strictly positive scalar curvature. Then using the Berger holonomy theorem [31] and the well known fact that metrics with holonomy $S U(m), S p(q), G_{2}$ or Spin(7) are Ricci flat we find that that they are only three posibilities for the holonomy group of $(N, h)$, namely $S O(2 m), U(m)$ and $S p(q) \cdot S p(1)$ when $m=2 q$. We will now treat each case separately.

If $\operatorname{Hol}(N, h)=S O(2 m)$, then if $m \neq 3$ the Lie algebra $\mathfrak{s o}(2 m)$ is simple and by lemma 6.1 we get easily that $\mathfrak{h}=\Lambda^{2}(H)$ so $(N, h)$ has constant sectional curvature by proposition 6.2. Hence $(N, h)$ is a symmetric space (in fact a round sphere), an absurdity. If $m=2$ then $\mathfrak{s o}(4)$ has two simple factors, each of dimension 3 . Thus if $\mathfrak{h} \neq \Lambda^{2}(H)$ it has dimension 3 and it follows that $\mathcal{V}$ has dimension 2 . We found that $(M, g, J)$ is 6-dimensional, Hermitian reducible nearly Kähler manifold, hence $(N, h)$ is symmetric by [1].

We consider now the case when $(N, h)$ has holonomy equal to $U(m)$. Then $(N, h)$ is a Kähler manifold with associated complex structure $I$. The holonomy bundle of $(N, h)$ is then $\Lambda^{1,1}(N)$. Denote by $\Lambda_{0}^{1,1}(N)$ the space of traceless two forms of type $(1,1)$. Using lemma 4.1 and the fact that $\mathfrak{h}$ has rank at least 2 we have either that $\mathfrak{h}=$ $\pi^{\star} \Lambda^{1,1}(N)$ or $\mathfrak{h}=\pi^{\star} \Lambda_{0}^{1,1}(N)$. As the curvature tensor of a Kähler Einstein manifold is determined by its restriction to $\Lambda_{0}^{1,1}(N)$ it is easy to conclude using proposition 6.2 that $(N, h)$ is symmetric, a contradiction.

Lastly, we suppose that $m=2 q$ and $\operatorname{Hol}(N, h)=S p(q) \cdot S p(1)$. Thus, $(N, h)$ is a quaternionic Kähler manifold and let us denote by $Q$ the 3-dimensional subbundle of $\Lambda^{2}(N)$ giving the quaternionic structure. Then we have a decomposition $\Omega^{2}(N)=$ $E_{1} \oplus E_{2} \oplus Q$ where fibers of $E_{1}$ and $Q$ are isomorphic in each point with $\mathfrak{s p}(q)$ and 
$\mathfrak{s p}(1)$ respectively. Furthermore, the holonomy bundle of the metric $h$ equals $E_{1} \oplus Q$. Now the curvature transformation of of the metric $h$ is determined by its restriction to $E_{1}$ as shown in [29]. Hence if it is constant on $E_{1}$, then $(N, h)$ is symmetric, in fact a quaternionic projective space. It follows that the only possibility is that $\mathfrak{h}=\pi^{\star} Q$ and this implies that $\mathcal{V}$ is of rank two. Thus, the canonical Hermitian connexion of $(M, g, J)$ has holonomy contained in $U(1) \times U(2 q)$ and we conclude by using a result from [24].

It remains to investigate the case when the manifold $(N, h)$ is a symetric space. We set the following :

DEFINITION 6.1. A simply connected, nearly Kähler manifold with special algebraic torsion and such that the canonical fibration has irreducible fiber is called homogenous of type $I V$ if the tensor $F$ has exactly two eigenvalues and the base space of the canonical fibration is a symmetric space.

Let us remark that the terminology "homogenous" is not yet justified. However, using the relation between the tensors $\bar{R}$ and $R^{h}$ given by O'Neill's relations, one easily gets that when $N$ is symmetric, $\bar{\nabla}$ is an Ambrose-Singer connection, hence $M$ is homogenous.

Now the proof of the theorem 1.1 is a consequence of the material previously presented.

7. Nearly Kähler metrics from Riemannian foliations. The main purpose of this section is to give a proof of the theorem 1.2, stated in the introduction. In order to do this let us consider a Kähler manifold $(M, g, J)$ toghether with a Riemannian foliation $\mathcal{F}$. Let $\mathcal{V}$ be the integrable distribution induced by $\mathcal{F}$. We recall that the metric $g$ is bundle-like with respect to our foliation, that is $\mathcal{L}_{V} g$ vanishes on $H$, the orthogonal complement of $\mathcal{V}$, for every $V$ in $\mathcal{V}$. As in the statement of the theorem 1.3 we make the assumption that the leaves of $\mathcal{F}$ are complex submanifolds of $M$, which gives that $J \mathcal{V} \subseteq \mathcal{V}$.

We consider now the Riemannian metric on $M$ defined by

$$
\hat{g}(X, Y)=\frac{1}{2} g(X, Y) \text { if } X, Y \in \mathcal{V}, \hat{g}(X, Y)=g(X, Y) \text { for } X, Y \text { in } H
$$

The metric $\hat{g}$ admits a compatible almost complex structure $\hat{J}$ given by $\hat{J}_{\mid \mathcal{V}}=-J$ and $\hat{J}_{\mid H}=J$. This almost complex structure was introduced in [6] for the case of twistor spaces over 4-manifolds.

Proposition 7.1. The manifold $(M, \hat{g}, \hat{J})$ is nearly Kähler. The distributions $\mathcal{V}$ and $H$ are parallel with respect to the canonical Hermitian connection of $(M, \hat{g}, \hat{J})$ which thus has reduced holonomy.

Proof. Let $A: T M \times T M \rightarrow T M$ be the O'Neill tensor of the Riemannian foliation induced by $\mathcal{V}$. As $g$ is Kähler we must have $A_{X} J=J A_{X}$ for all $X$ in $T M$. Using the relations between the Levi-Civita connections of $\hat{g}$ and $g$ given in [27] we obtain after a standard computation :

$$
\begin{aligned}
& \left(\hat{\nabla}_{X} \hat{J}\right) V=-\left(\hat{\nabla}_{V} \hat{J}\right) X=-A_{X}(J V) \\
& \left(\hat{\nabla}_{V} \hat{J}\right) W=0,\left(\hat{\nabla}_{X} \hat{J}\right) Y=2 A_{X}(J Y)
\end{aligned}
$$

for every $X, Y$ in $\mathcal{V}$ and $V, W$ in $H$. It is now straightforward to conclude $\square$ 
COROLLARY 7.1. The twistor space of a positive quaternionic-Kähler manifold of dimension $4 k$ admits a canonical NK structure with reducible holonomy, contained in $U(1) \times U(2 k)$.

Proof. We have only to recall [29] that such a twistor space is the total space of a Riemannian submersion with totally geodesic fibers of dimension 2 and that it admits a compatible Kähler structure $\square$

REMARK 7.1. If one drops the condition that the distibution $\mathcal{V}$ be totally geodesic, the construction above produces only a quasi-Kähler manifold.

We are now going to prove theorem 1.2. We remark that the nearly Kähler manifold $(M, \hat{g}, \hat{J})$ has the property that $\mathcal{V} \bullet \mathcal{V}=0$ and $H \bullet H \subseteq \mathcal{V}$. Using the decomposition result of the proposition 2.1 we obtain that (under the simple connectivity assumption) $M$ is the Riemannian product of a Kähler manifold and a nearly Kähler manifold with special algebraic torsion. Hence by the structure results of sections 4,5 and 6 the de deRham decomposition of the second factor consists only of spaces of type III and IV and twistor spaces over positive quaternionic Kähler manifolds equipped with their canonical nearly Kähler metric. The proof of the theorem comes now by reversing the construction done in the beginning of the section, and by using proposition 4.3. However, we postpone until the next section the proof of the fact that homogeneous space $(M, g, J)$ of type III or IV is a homogeneous Kähler manifold when endowed with the metric $\bar{g}$ and the complex structure $\bar{J}$.

8. Homogeneous nearly Kähler manifolds. In this section we will investigate some properties of homogeneous nearly Kähler manifolds that are introduced by the following definition.

DEFINITION 8.1. Let $\left(M^{2 n}, g, J\right)$ a strict and complete nearly Kähler manifold. It is called homogeneous if it admits a transitive action of a connected, closed subgroup of the group of holomorphic isometries of $(g, J)$.

Let us now prove that what we called homogenous spaces of type I, II, III, IV are in fact homogeneous nearly Kähler manifolds and that for the spaces of type III and IV the natural Kähler metric is homogeneous.

Proposition 8.1. Let $\left(M^{2 n}, g, J\right)$ be a strict and complete nearly Kähler manifold.

(i) If $\bar{\nabla} \bar{R}=0$ then $(M, g, J)$ is a homogeneous nearly Kähler space. In particular $M$ is naturally reductive and if moreover, $M$ is simply connected, then $(M, g, J)$ is a Riemannian 3-symmetric space.

(ii) If $(M, g, J)$ is a space of type III or IV then $(M, \bar{g}, \bar{J})$ is a homogeneous Kähler manifold.

Proof. (i) In the terminology of [28], the $\bar{\nabla}$-parallel tensor $T_{X} Y=-\frac{1}{2}\left(\nabla_{X} J\right) J Y$ is a homogeneous structure. The theorem of Ambrose and Singer (see [28]) gives now an explicit construction of $M$ as a homogeneous space and from this it is easy to conclude using a result of Gray (see [13], proposition 5.5, page 358).

(ii) Using the relation between the Levi-Civita of $\bar{g}$ and $\bar{\nabla}$ given in the proof of proposition 4.3 one can prove directly that the tensor $T_{X} Y$ below is a homogeneous structure for $(M, \bar{g}, \bar{J})$. We omit the details.

Let us give now a corollary of theorem 1.1 related to homogeneous nearly Kähler spaces.

Proposition 8.2. Let $\left(M^{2 n}, g, J\right)$ be a homogeneous nearly Kähler manifold which is also strict and simply connected. Then $(M, g, J)$ is isometric and biholomor- 
phic to a Riemannian product whose factors belongs to the following list :

-homogeneous 6-dimensional

-spaces of type I,II,III or IV

-twistor spaces over positive quaternionic Kähler manifolds which are homogeneous as nearly Kähler manifolds.

Proof. It is not hard to see that each of the manifolds occuring in the decomposition of theorem 1.1 is irreducible in the usual Riemannian sense. Hence, that decomposition is the deRham decomposition of the nearly Kähler manifold $(M, g, J)$ and by a result of Hano [21] it follows that the connected isometry group of $(M, g)$ is the direct product of the isometry groups of the factors. But this implies that each factor is a homogeneous space and using that $J$ preserves our decomposition it is straightforward to conclude. $\mathrm{T}$

The rest of this section will be devoted to the proof of the following classification result.

THEOREM 8.1. Let $\left(M^{2 n}, g, J\right)$ be a homogeneous nearly Kähler manifold which is also strict and simply connected. Then $(M, g, J)$ is isometric and bihomorphic to a Riemannian product whose factors belong to the following list :

-homogeneous 6-dimensional nearly Kähler manifolds -spaces of type I,II,III or IV

Before getting into the proof let us make some comments on theorem 8.1. As any Riemannian 3-symmetric space is nearly Kähler as soon as it is naturally reductive the previous result yields a geometric version of the classification of naturally reductive 3 -symmetric spaces. Note that the general classification theory of 3-symmetric spaces develloped in [13] was obtained using quite involved Lie algebra arguments. It also follows from theorem 8.1 that Wolf\&Gray's conjecture (see [39], page 158) asserting that any strict homogenenous nearly Kähler manifold is 3-symmetric holds true if and only if it holds in 6-dimensions. Concerning the types of homogenenous spaces appearing in the theorem let us recall that those of type I are compact, simply connected isotropy irreducible homogeneous spaces, of naturally reductive type, carrying a nearly Kähler structure. Here our geometric results do not allow classification. For spaces of type II, further work is required to localize completely this class in Gray's list (see section 3, remark 3.1). Finally, spaces of types III and IV can be classified using on the one hand the classification of compact and simply connected, irreducible Hermitian symmetric spaces and other the other hand the description of the fiber of the canonical fibration in the terms of the Lie algebra $\mathfrak{h}$ together with lemma 6.1 (see [25] for details). Note that the spaces of type III and IV were already studied from various points of view in [30] and [4].

Let us now prove the theorem 8.1. It suffices to show that if a twistor space with its canonical nearly Kähler metric is homogeneous then the base quaternionic manifold is symmetric. For, let us consider such a twistor space; it is a strict nearly Kähler manifold with special algebraic torsion $(M, g, J)$ with decomposition $T M=\mathcal{V} \oplus H$ where $\mathcal{V}$ is of rank two and such the tensor $F$ has a single eigenvalue of the form $\frac{k}{4}, k>0$.

Let us set now the following notation. If $(Z, h)$ is a Riemannian manifold we denote by $\iota(Z, h)$ the Lie algebra of Killing vector fields. Furthermore, if $I$ is an almost complex structure giving $(Z, h)$ the structure of a almost complex manifold we define $\iota(Z, h, J)$ to be the Lie algebra of holomorphic Killing vector vector fields. 
Our aim is to obtain a comparison result between $\iota(M, \bar{g}, \bar{J})$ and $\iota(M, g, J)$. Let $X=V+K$ be an element of $\iota(M, g, J)$ split into its horizontal and vertical components. We use the parallelism of the decomposition $T M=\mathcal{V} \oplus H$ with respect to the connection $\bar{\nabla}$ in order to see that the Killing equation for $X$ (the skew-symmetry of $\nabla X)$ is equivalent with the following system of equations for its components :

$$
\begin{aligned}
& <\bar{\nabla}_{Y} K, Z>+<\bar{\nabla}_{Z} K, Y>=0 \\
& <\bar{\nabla}_{W} K, Y>+<\bar{\nabla}_{Y} V, W>=0 \\
& <\bar{\nabla}_{W_{1}} V, W_{2}>+<\bar{\nabla}_{W_{2}} W_{1}>=0
\end{aligned}
$$

where $Y, Z$ are in $H$ and $W, W_{1}, W_{2}$ are in $\mathcal{V}$. Using the same arguments the condition that $X$ be a $J$-holomorphic vector field projets onto the following two systems :

$$
\begin{aligned}
& \bar{\nabla}_{J Y} K-J\left(\bar{\nabla}_{Y} K\right)=-2\left(\nabla_{Y} J\right) V \\
& \bar{\nabla}_{J Y} V-J\left(\bar{\nabla}_{Y} V\right)=-2\left(\nabla_{Y} J\right) K
\end{aligned}
$$

and

$$
\begin{aligned}
& \bar{\nabla}_{J W} K-J\left(\bar{\nabla}_{W} K\right)=-2\left(\nabla_{W} J\right) K \\
& \bar{\nabla}_{J W} V-J\left(\bar{\nabla}_{W} V\right)=0
\end{aligned}
$$

whenever $Y$ and $W$ are in $H$ and $\mathcal{V}$ respectively.

REMARK 8.1. Neither all the equations of the systems (8.1), (8.2), (8.3) are independent, nor all will be used in what follows. We gave all of them for completeness reasons.

We will need now the following auxiliary result.

LEMMA 8.1. If $Y$ and $W$ are in $H$ and $\mathcal{V}$ respectively, then:

(i) $\bar{\nabla}_{W} V=\frac{f}{2} J W$ where $f=d^{\star}(J V)$,

(ii) $\bar{\nabla}_{W} K=\left(\nabla_{W} J\right) J K$,

(iii) $\bar{\nabla}_{Y} V=\left(\nabla_{Y} J\right) J K$.

Proof. The proof of (i), an easy exercise, will be left to the reader. In order to prove (ii) let $\left\{e_{i}\right\}, i=1,2$ be a local orthonormal basis in $\mathcal{V}$. Using the second equation of (8.1) with $W=e_{i}$ and next deriving it in the direction of $e_{i}$ we obtain :

$$
<\bar{\nabla}_{e_{i}} \bar{\nabla}_{e_{i}} K, Y>+<\bar{\nabla}_{e_{i}} K, \bar{\nabla}_{e_{i}} Y>+<\bar{\nabla}_{e_{i}} \bar{\nabla}_{Y} V, e_{i}>+<\bar{\nabla}_{Y} V, \bar{\nabla}_{e_{i}} e_{i}>=0 .
$$

Using again the second equation of (8.1) for the second and third terms of the previous sum we get that $<\bar{\nabla}_{e_{i}, e_{i}}^{2} K, Y>+<\bar{\nabla}_{e_{i}, Y}^{2} V, e_{i}>=0$. But by the Ricci identity $\bar{\nabla}_{e_{i}, Y}^{2} V=\bar{\nabla}_{Y, e_{i}}^{2} V-\bar{R}\left(e_{i}, Y\right) V+\bar{\nabla}_{\left(\nabla_{Y} J\right) J e_{i}} V$ and moreover $\bar{R}\left(e_{i}, Y\right) V=0$ as $e_{i}$ is in $\mathcal{V}$ and $Y$ belongs to $H$. Now ,

$$
<\bar{\nabla}_{Y, e_{i}}^{2} V, e_{i}>=<\bar{\nabla}_{Y} \bar{\nabla}_{e_{i}} V-\bar{\nabla}_{\bar{\nabla}_{Y} e_{i}} V, e_{i}>=\frac{1}{2} Y . f<J e_{i}, e_{i}>=0
$$

by (i). Hence $<\bar{\nabla}_{e_{i}, e_{i}}^{2} K, Y>+<\bar{\nabla}_{\left(\nabla_{Y} J\right) J e_{i}} V, e_{i}>=0$ and using the second equation of (8.1) (together with the fact that $\nabla_{e_{i}} J$ is skew-symmetric and $J$-anticommuting) we arrive at $\left\langle\bar{\nabla}_{e_{i}, e_{i}}^{2} K, Y>=<\left(\nabla_{e_{i}} J\right) J \bar{\nabla}_{e_{i}} K, Y>\right.$. We define now

$$
S=\sum_{i=1}^{2}\left(\nabla_{e_{i}} J\right) J \bar{\nabla}_{e_{i}} K
$$


Then $S=\sum_{i=1}^{2}\left(\nabla_{J e_{i}} J\right) J \bar{\nabla}_{J e_{i}} K$ and we use that $\left(\nabla_{J U} J\right) J T=-\left(\nabla_{U} J\right) T$ for all $U, T$ in $T M$ and also the first equation of (8.3) to show that $S=\sum_{i=1}^{2}\left(\nabla_{e_{i}} J\right)^{2} K=-\frac{k}{2} K$. Resuming, we have that $\sum_{i=1}^{2}\left\langle\bar{\nabla}_{e_{i}, e_{i}}^{2} K, Y\right\rangle=-\frac{k}{2}\langle K, Y\rangle$. On the other hand, as $\bar{\nabla}_{e_{i}} e_{i}=\nabla_{e_{i}} e_{i}$ and by the second equation of the system (8.1) we obtain that

$$
\begin{aligned}
& \left\|\bar{\nabla}_{e_{i}} K\right\|^{2}=e_{i} \cdot<\bar{\nabla}_{e_{i}} K, K>-<\bar{\nabla}_{\nabla_{e_{i}} e_{i}} K, K>-<\bar{\nabla}_{e_{i}, e_{i}}^{2} K, K>= \\
& -\left[e_{i} \cdot<\bar{\nabla}_{K} V, e_{i}>-<\bar{\nabla}_{K} V, \nabla_{e_{i}} e_{i}>\right]-<\bar{\nabla}_{e_{i}, e_{i}}^{2} K, K>.
\end{aligned}
$$

As the vector field $\bar{\nabla}_{K} V$ is vertical, we obtain easily that $\sum_{i=1}^{2}\left\|\bar{\nabla}_{e_{i}} K\right\|^{2}=\frac{k}{2}\|K\|^{2}+d^{\star} \alpha$ where $\alpha$ is the 1 -form of $M$ dual to $\bar{\nabla}_{K} V$. By the previous considerations, this implies that

$$
\sum_{i=1}^{2}\left\|\bar{\nabla}_{e_{i}} K-\left(\nabla_{e_{i}} J\right) J K\right\|^{2}=d^{\star} \alpha
$$

and the conclusion follows by integration over $M$. The identity in (iii) is a consequence of (i) and of the second equation of (8.1).

We can now prove the following.

Proposition 8.3. We have $\iota(M, g, J)=\iota(M, \bar{g}, \bar{J})$.

Proof. Let us prove that $\iota(M, g, J) \subseteq \iota(M, \bar{g}, \bar{J})$. If $\widetilde{\nabla}$-denotes the LeviCivita connection of $(M, \bar{g})$ then we recall that $\widehat{\nabla}_{X} Y=\nabla_{X} Y, \widetilde{\nabla}_{X} V=\bar{\nabla}_{X} V-$ $\left(\nabla_{X} J\right) J V, \widetilde{\nabla}_{V} X=\bar{\nabla}_{V} X$ and $\widetilde{\nabla}_{V} W=\nabla_{V} W$ for all $X, Y$ in $H$ and $V, W$ in $\mathcal{V}$ respectively. For, if $X$ in $\iota(M, g, J)$ it is easy to see, using the previous lemma that the equation $\widetilde{\nabla} X=0$ follows from the system 8.1 (of course we have to take into account that on a compact Kähler manifold every Killing vector field is holomorphic). The other inclusion can be proven, for example, using the same technique, and will be left to the reader.

It follows that $\operatorname{Isom}^{0}(M, g, J)=\operatorname{Isom}^{0}(M, \bar{g}, \bar{J})$, hence if $(M, g, J)$ is a homogeneous nearly Kähler manifold then $(M, \bar{g}, \bar{J})$ is a homogeneous Kähler manifold. Recall now that being a twistorial space $(M, \bar{g}, \bar{J})$ has a canonical complex structure (see [29]) and this is not hard to see that it is in fact a homogeneous complex contact manifold. It follows then by the results in [38] that the base quaternionic-Kähler manifold is in fact symmetric and this ends the proof of theorem 8.1.

\section{REFERENCES}

[1] F. Belgun , A. Moroianu, Nearly Kähler 6-manifolds with reduced holonomy, Ann. Glob. An. Geom., 19 (2001), pp. 307-319.

[2] A. BESSE, Einstein manifolds, Springer-Verlag, New York, 1987.

[3] C.P. Boyer, K. Galicki, B.M. ManN, E.G. Rees, Compact 3-sasakian manifolds 7-manifolds with arbitrary second Betti number, Inv. Math., 131 (1998), pp. 321-344.

[4] F. E. Burstall, J. H. RaWnsley, Twistor theory for Riemannian symmetric spaces, Lect. Notes In Math. 1424, 1990.

[5] R. Cleyton, G-structures and Einstein metrics, Ph.D. thesis, Odense, 2001. 
[6] J. Eels, S. Salamon, Constructions twistorielles des applications harmoniques, C. R. Acad. Sc. Paris, 296 (1983), pp. 685-687.

[7] M. Falcitelli, A.M PASTORE, A note on almost Kähler and nearly Kähler submersions, J. Geom., 69 (2000), pp. 79-87.

[8] TH. Friedrich, I. Kath, A. Moroianu, U. Semmelmann, On nearly parallel $G_{2}$-structures, J. Geom. Phys., 23 (1997), pp. 259-286.

[9] TH. FriedRICH, Weak Spin(9)-structures on 16-dimensional manifolds, Asian J. Math., 5 (2001), pp. 129-160.

[10] Th. Friedrich, S. IVANOV, Parallel spinors and connections with skew-symmetric torsion in string theory, preprint 2001, eprint arXiv:math. DG/0102142.

[11] A. GraY, Nearly Kähler manifolds, J. Diff. Geom., 4 (1970), pp. 283-309.

[12] A. GrAY, Weak holonomy groups, Math. Z, 125 (1971), pp. 290-300.

[13] A. GRAY, Riemannian manifolds with geodesic symmetries of order 3, J. Diff. Geometry, 7 (1972), pp. 343-369.

[14] A. GRAY, The structure of nearly Kähler manifolds, Math. Ann., 223 (1976), pp. 233-248.

[15] A. Gray, L. M. Hervella, The sixteen classes of almost Hermitian manifolds and their linear invariants, Ann. Mat. Pura Appl., 123 (1980), pp. 35-58.

[16] R. Grunewald, Six-Dimensional Riemannian manifolds with real Killing spinors, Ann. Global Anal. Geom., 8 (1990), pp. 43-59.

[17] S. HeLGason, Differential geometry, Lie groups, and symmetric spaces, Academic Press, 1978.

[18] D.L. Johnson, Kähler submersions and holomorphic connections, J. Diff. Geom., 15 (1980), pp. $71-79$.

[19] V.F. Kirichenko, K-spaces of maximal rank, Mat. Zametki, 22, no.4 (1977), pp. 465-476.

[20] S. KовAYASHI, On compact Kähler manifolds with positive definite Ricci tensor, Ann. of Math., 74 (1961), pp. 570-574.

[21] S. KobaYASHI, K. Nomizu, Foundations of differential geometry, vol.1, Wiley, 1963.

[22] C. Lebrun, S. Salamon, Strong rigidity of positive quaternion-Kähler manifolds, Invent. Math., 118 (1994), pp. 109-132.

[23] C. Lebrun, Fano manifolds, contact structures and quaternionic geometry, International J. Math., 6 (1995), pp. 419-437.

[24] P.A. NAGY, On nearly Kähler geometry, Ann. Glob. An. Geom., 22 (2002), pp. 167-178.

[25] P.A. NAGY, Homogeneous nearly Kähler manifolds, in preparation, 2002.

[26] Y. S. POON, S. SALAMON, Eight-dimensional quaternion Kähler manifolds with positive scalar curvature, J. Diff. Geom., 33 (1990), pp. 363-378.

[27] PH. TondeUr, Geometry of Foliations, Birkhäuser Verlag, 1997.

[28] F. Tricerri and L. VANHECKE, Homogenous structures on Riemannian manifolds, London Math. Soc. Lecture Notes, 83 (1982).

[29] S. SAlamon, Quaternionic Kähler manifolds, Invent. Math., 67 (1982), pp. 143-171.

[30] S. SALAmon, Harmonic and holomorphic maps, In : Seminar L. Bianchi, Lect. Notes In Math. $1164,1986$.

[31] S. SALAMON, Riemannian geometry and holonomy groups, Longman Scientific \&Technical, 1989.

[32] S. Salamon, Almost parallel structures, to appear in Contemp. Math., eprint arXiv:math.DG/0107146

[33] K. SeKigawa, L. VANHECKe, Almost hermitian manifolds with vanishing first Chern classes or Chern numbers, Rend. Sem. Mat. Univ. Pol. Torino, 50 (1992), pp. 195-208.

[34] R. W. SHARPE, Differential geometry, Springer Verlag, 1997.

[35] A. SWANN Weakening holonomy, Proceedings of the second meeting on quaternionic structures, World Sientific, Singapore (2001), pp. 405-415.

[36] Y. Watanabe, K. Takamatsu, On a $K$-space of constant holomorphic sectional curvature, Kodai Math. Sem. Rep., 25 (1973), pp. 297-306.

[37] B. Watson, Almost hermitian submersions, J. Diff. Geom., 11 (1976), pp. 147-165.

[38] J.A. Wolf, Complex homogeneous contact manifolds and quaternionic symmetric spaces, J.Math.Mech., 14, No.6 (1965), pp. 1033-1047.

[39] J.A. Wolf, A. GRAY, Homogeneous spaces defined by Lie group automorphisms I, II, J. Diff. Geom., 2 (1968), pp. 77-114, pp. 115-59. 